\title{
How Chemical Environment Activates Anthralin and Molecular Oxygen for Direct Reaction
}

Emerald S. Ellis, Luke T. MacHale, Robert K. Szilagyi, ${ }^{*}$ Jennifer L. DuBois*

Department of Chemistry and Biochemistry, Montana State University, Bozeman, MT 5971

\section{COMPUTATIONAL DETAILS}

In addition to the tables and figures below, a zip file, downloadable from the JOC website, is available containing all computational results, including validation of the level of theory, molecular structures, and analysis spreadsheets that are in support of our experimental observations of spontaneous reactivity of anthralin/dithranol molecule with molecular oxygen without any catalyst or co-substrate. The same information is available at the free, publically accessible Zenodo dataset depository, DOI: 10.5281/zenodo.3554508. This information includes:

${ }^{* * * *}$ Free volume correction:

We employed a correction to translational entropy as suggested by Whitesides et al. in their paper entitled "Estimating the Entropic Cost of Self-Assembly of Multi-particle Hydrogen-Bonded Aggregates", JOC 1998, 64, 3821-3830.

In addition to the worked out example in the publication (for chloroform) we provided parameters for all the considered solvents and beyond.

****Theory validation:

We used the computational work of Korth and Mulder (JOC 2013, 78, 7674) for a high level wave function reference calculations, in order to validate the various level of density functional for carrying out the large scale computational mapping of tautomerism in anthralin/dithranol. All computational results and the detailed analysis are provided in the archive.

In addition to the relative stability of the gas phase structures, a brief inquiry was completed for critically assessing the various polarizable continuum model.

Remarkably, the most accurate functional and the most adequate polarizable continuum model were found to come from the same theoretical and computational chemistry school at University of Minneapolis (Truhlar/Cramer). 


\section{${ }^{* * * *}$ Molecular structures}

We provide the atomic positional coordinates of all the tautomers in their equilibrium structures along with their ground state energies (list of energies.txt file) that are mentioned in the manuscript or the JOC supporting information.

\section{${ }^{* * * *}$ Input files}

The folder contains all the input files used to generate the data reported in the manuscript. The files are organized by solvents in separate folders.

\section{TABLES}

Table S1

Summary of solvent/amino acid side chain correspondence and solvent parameters used from Universal Solvation Model Based on Solute Electron Density (SMD $\left.\operatorname{model}^{1}\right)$.

Table $52 \quad$ Errors in tautomerisation enthalpies $\left(\Delta \Delta^{\mathrm{t}} \mathrm{H}, \mathrm{kJ} \mathrm{mol}^{-1}\right)$ calculated using various density functionals relative to the corresponding CBS-QB3 reference values ${ }^{2}$ for equilibrium structures of gas phase isomers for 1,8-dihydroxyanthrone (keto) and 1,8,9trihydroxy-anthracane (enol) molecules.

SI-8

Table S3 Summary of nuclear repulsion ( $E_{\text {nuc }}$, a.u.), total electronic energy ( $E_{S C F}$, a.u.), enthalpy (H, a.u.), uncorrected (G, a.u.) and corrected ( $G^{\text {corr }}$, a.u.) Gibbs free energy, condensed phase translational entropy $\left(\mathrm{J} \mathrm{mol}^{-1} \mathrm{~K}^{-1}\right.$, at $100 \mu \mathrm{M}$ concentration, $\mathrm{T}=298$ K) calculated at MN15/6-311++G**/SMD(solvent) level of theory.

Table S4 Worksheet for correcting gas phase translational entropy (according to Whitesides et al. ${ }^{3}$ ) to consider condensed phase free volume of in $100 \mu \mathrm{M}$ solution of dithranol and its isomers in the evaluated solvents.

SI-10

Table S5 Summary of UV/visible absorbance data for dithranol and the oxygenation product dithranone: Wavelengths ( $\mathrm{nm}$ ) of peak maxima and shoulders.

SI-11

Table S6 Summary of fluorescence data for dithranol: Wavelengths (nm) of peak maxima and shoulders. The upper section summarizes dithranol fluorescence emission spectra while the lower section summarizes fluorescence excitation spectra, where light emission at $495 \mathrm{~nm}$ was monitored.

$\mathrm{SI}-12$

\section{SCHEMES}

Scheme S1: $\quad$ Overview of tautomers with key $\mathrm{C}-\mathrm{H}$ and $\mathrm{O}-\mathrm{H}$ positional variations and representative hydrogen bonding patterns.

SI-13 
Figure S1 The rate of dithranol oxidation in air varies with solvent according to the initial fraction of enolate present, as illustrated by time resolved UV/Vis spectroscopy and HPLC. Reactions of dithranol in air (room temperature, 100 rpm shaking, airsaturation) were monitored over time by UV/vis absorbance in (A) $\mathrm{CHCl}_{3}(124 \mu \mathrm{M}$ dithranol) and (B) DMF (110 $\mu \mathrm{M}$ dithranol). (B, inset) Discontinuous HPLC was used to monitor the disappearance of dithranol (white squares) and appearance of dithranone (blue circles) over time in DMF. The data were fit to single exponential curves yielding $\mathrm{k}=0.15 \mathrm{~h}$ (dithranol disappearance) or $\mathrm{k}=0.24 \mathrm{~h}$ (dithranone appearance). (C-E) UV/vis absorbance spectra of the oxygenation product, dithranone $(50 \mu \mathrm{M})$, in (C) $\mathrm{CHCl}_{3}$, (D) DMF, and (E) $2 \mathrm{ME}$.

$\mathrm{SI}-14$

Figure S2 NMO lowers the pKa of dithranol by greater than $2 \mathrm{pH}$ units. Spectrophotometric $\mathrm{pH}$ titrations of (A) $46 \mu \mathrm{M}$ dithranol and (B) $39 \mu \mathrm{M}$ dithranol mixed with $80 \mu \mathrm{M}$ NMO (NMO-dithranol complex) were performed discontinuously using a series of aqueous buffers containing $100 \mathrm{mM}$ buffer and $300 \mathrm{mM} \mathrm{NaCl}$ pre-adjusted to $\mathrm{pH} 5.02,5.53$, $5.97,6.50,7.00,7.57,8.06,8.43,8.90,9.54,9.95,10.47,11.15$ (see Solvents and Reagents section of Experimental). An approximate isosbestic point at $345 \mathrm{~nm}$ is observed in panel A. An isosbestic point at $368 \mathrm{~nm}$ is observed in panel $B$. Upon binding to the enzyme, the absorbance maxima in dithranol's high-pH spectrum are observed to red shift by approximately $8 \mathrm{~nm}$. The individual peaks, especially above $400 \mathrm{~nm}$, are more well-resolved. (Insets) $\mathrm{pK}_{a}$ values were determined by plotting the absorbance at $385 \mathrm{~nm}$ (dithranol) or $394 \mathrm{~nm}$ (NMO-dithranol complex) as a function of $\mathrm{pH}$ and fitting the data to a sigmoidal curve. The active site pocket of NMO lowers the $\mathrm{p} K_{\mathrm{a}}$ of dithranol from $9.28 \pm 0.07$ to $7.15 \pm 0.09\left(\Delta \mathrm{p} K_{\mathrm{a}}=2.13 \pm 0.11\right) . \quad \mathrm{SI}-15$

Figure S3 Gibbs free energy landscape (corrected for translational entropy in $100 \mu \mathrm{M}$ solution at MN15/6-311++G**/SMD level of theory) of dithranol tautomers as defined in Scheme S1. All energy values are given relative to the lowest energy keto isomer in gas phase. Isomers are ordered according to their relative energies in gas phase as shown on the left-hand side of the figure. Enolate structures were mapped considering protonated solvents at infinite separation and both embedded in polarizable continuum model SMD.

SI-16

Figure S4 The UV/vis absorbance spectrum of dithranol is solvent-dependent. Representative spectra of $50 \mu \mathrm{M}$ dithranol in (A) $\mathrm{CHCl}_{3}$, (B) benzene, (C) $\mathrm{H}_{2} \mathrm{O}(25$ $\mu \mathrm{M}$ dithranol) (D) 2ME, (E) DMSO, and (F) DMF. In $\mathrm{CHCl}_{3}$ and benzene, the spectrum of dithranol exhibits a single broad peak centered at 358 and $359 \mathrm{~nm}$, 
respectively, which is similar to that observed in aqueous buffer at low $\mathrm{pH}$ ( $<1$ unit from the $\mathrm{pK}_{\mathrm{a}}$ ). (See Figure S2A.) The spectrum in pure distilled water (C) resembles the spectra shown in (A) and (B); however, the source of asymmetry in this spectrum is unclear. In DMF, dithranol's spectrum is similar to the NMO-dithranol spectrum measured in high $\mathrm{pH}\left(>1\right.$ unit from $\mathrm{pK}_{\mathrm{a}}$ ) aqueous buffer: dominated by a sharp peak at $391 \mathrm{~nm}$ with a shoulder at $373 \mathrm{~nm}$ and three minor peaks at 428, 453, and $479 \mathrm{~nm}$. (See Figure S2B.) The bathochromic shift and appearance of additional peaks at higher wavelengths are indicative of increased conjugation (e.g., see spectra for benzene, naphthalene, and anthracene, representing increases in conjugation) consistent with the assignment of a ketone isomer to the single-peak spectrum and a more-conjugated, enol(ate) isomer to the multi-peak, red-shifted spectrum. In DMSO and $2 \mathrm{ME}$, dithranol's spectrum exhibits features of both $\mathrm{pH}$-extremes, suggesting that dithranol exists as a mixture of the two species in these solvents.

SI-17

Figure S5 The absorbance spectra of dithranol in neat DMSO or 2ME can be simulated as linear combinations of spectra measured in acidic or alkaline solvent. UV/Vis absorption spectra of dithranol at varying concentrations were measured in DMSO or $2 \mathrm{ME}$ in the presence of excess $(1 \mathrm{mM}) \mathrm{HCl}$ or $\mathrm{NaOH}$. Simulated absorbance spectra were generated as linear combinations of the two component spectra representing the protonated ketone and deprotonated enolate forms of dithranol. Molar absorptivity is plotted as a function of wavelength for dithranol in (A) $\mathrm{DMSO}_{\text {Acidic }}$ (blue) or $\mathrm{DMSO}_{\text {Alk }}$ (red) and (B) $2 \mathrm{ME}_{\text {Acidic }}$ (blue) or $2 \mathrm{ME}_{\text {Alk }}$ (red). (C) The absorbance spectrum of $50 \mu \mathrm{M}$ dithranol in neat DMSO (black) and a simulated spectrum (pink/dashed) that was generated as the sum of the weighted component spectra shown in blue (73\%) and red (27\%). (D) Absorbance spectrum of $50 \mu \mathrm{M}$ dithranol in $2 \mathrm{ME}$ (black) and a simulated spectrum (pink/dashed) that was generated as the sum of the weighted component spectra shown in blue (76\%) and red $(24 \%)$.

SI-18

Figure S6 The fluorescence emission intensity and excitation spectra of dithranol show different solvent-dependent trends. Excitation spectra were measured by illuminating dithranol over a range of wavelengths (lighter-colored lines, 250-470 nm) and monitoring emitted fluorescence at $495 \mathrm{~nm}$. The emission spectra (darker lines, 400-650 $\mathrm{nm}$ ) were then produced by exciting dithranol at the visible wavelength at which maximum fluorescence intensity was observed in the excitation spectrum $\left(\lambda_{\mathrm{ExC}}\right)$. Representative spectra are shown in (A) $\mathrm{CHCl}_{3}\left(50 \mu \mathrm{M}, \lambda_{\mathrm{Exc}}=380 \mathrm{~nm}\right)$, (B) benzene $\left(50 \mu \mathrm{M}, \lambda_{\mathrm{Exc}}=380 \mathrm{~nm}\right)$, (C) $\mathrm{H}_{2} \mathrm{O}\left(25 \mu \mathrm{M}, \lambda_{\mathrm{Exc}}=383 \mathrm{~nm}\right)$, (D) $2 \mathrm{ME}(5 \mu \mathrm{M}$, $\left.\lambda_{\mathrm{ExC}}=389 \mathrm{~nm}\right)$, (E) DMSO $\left(5 \mu \mathrm{M}, \lambda_{\mathrm{Exc}}=393 \mathrm{~nm}\right)$, and (F) DMF $\left(5 \mu \mathrm{M}, \lambda_{\mathrm{Exc}}=391 \mathrm{~nm}\right)$. 
Little to no fluorescence was observed in solutions of dithranol in $\mathrm{CHCl}_{3}$ and benzene. By contrast, dithranol fluoresces orders of magnitude more intensely in DMF than any other neat solvent. (Note the differences in the various $y$-scales.) Intermediate fluorescence intensity is observed in neat DMSO or 2ME, which can be increased by addition of $\mathrm{NaOH}$ (not shown). Keto forms (Scheme S1) lacking conjugation in the center ring are expected be flexible, diminishing conjugation in the 3 -ring system and consequently lowering fluorescence. The intense transitions in dithranol's fluorescence emission spectrum in DMF are consistent with a fully conjugated, planar 3-ring system. The fluorescence spectra of dithranol in DMSO and $2 \mathrm{ME}$ are of intermediate intensity, reflecting the mixed ketone/enol(ate) composition. The small amount of fluorescence observed in solutions of dithranol in $\mathrm{CHCl}_{3}$ and benzene could suggest that a very small fraction of dithranol exists as a planar enol in those solvents. This is supported by the shape of the fluorescence excitation spectra $(200-450 \mathrm{~nm})$ when emission at $495 \mathrm{~nm}$ is monitored. This has a maximum near $380 \mathrm{~nm}$ with small peaks at higher wavelengths, rather than a single broad peak near $360 \mathrm{~nm}$ as in the absorbance spectrum for the ketone, which would be expected if the ketone isomer itself was minimally fluorescent, e.g. while transiently planar. This blue-shift in maximum excitation wavelength is accompanied by a blue-shift in the entire emission spectrum, which suggests that it belongs to a dithranol isomer other than the keto or enolate.

$\mathrm{SI}-18$

Figure S7 ${ }^{1} \mathrm{H}-\mathrm{NMR}$ spectra of $10 \mathrm{mM}$ dithranol $\left(\mathrm{A} ; \mathrm{CDCl}_{3}, 600 \mathrm{MHz}\right.$ ) and $18 \mathrm{mM}$ dithranone (B; $\left.\left(C_{3}\right)_{2} \mathrm{SO}, 600 \mathrm{MHz}\right)$. In chloroform, the spectrum for dithranol (A) exhibits four sets of peaks, which integrate to a total of ten protons, consistent with the keto isomer of dithranol as shown. The presence of a sharp singlet peak at $12.3 \mathrm{ppm}$, which integrates to two protons, suggests that the two potentially exchangeable hydroxyl protons are not labile, but rather, involved in stable $\mathrm{H}$-bonds ${ }^{4}$ with the carbonyl oxygen on the central ring, as observed in the crystal structure (Scheme S1, 2HB-keto structure). Two protons are represented by a triplet and four by doublet-ofdoublet peaks in the region typically associated with aromaticity (6-8 ppm), highlighted in the dashed box and shown on an expanded scale in the inset. An upfield peak at $4.4 \mathrm{ppm}$ represents the two protons of the methylene carbon of the central ring, and their presence is unique to the keto isomers. Dithranol ${ }^{1} \mathrm{H}-\mathrm{NMR}$ summary $\left(\mathrm{CDCl}_{3}, 600 \mathrm{MHz}\right): \delta_{\mathrm{H}} 12.31(2 \mathrm{H}, \mathrm{s}), 7.51\left(2 \mathrm{H}^{*}, \mathrm{t}, J=7.9\right), 6.93(4 \mathrm{H}, \mathrm{dd}, J=$ 1.6, 0.5), $4.38(2 \mathrm{H}, \mathrm{s})$. In DMSO, the NMR spectrum of dithranone $(B)$ exhibits four peaks, which integrate to a total of eight protons. The hydroxyl protons are 
represented by a sharp singlet peak at $11.9 \mathrm{ppm}$ which integrates to two protons, suggesting that these are involved in stable $\mathrm{H}$-bonds ${ }^{4}$ with the carbonyl oxygen on the central ring, as observed in dithranol. Two protons are represented by a triplet and four by doublet-of-doublet peaks in the region typically associated with aromaticity (dashed box, shown on an expanded scale in the inset). Dithranone ${ }^{1} \mathrm{H}$ NMR summary $\left(\left(\mathrm{CD}_{3}\right)_{2} \mathrm{SO}, 600 \mathrm{MHz}\right): \delta_{\mathrm{H}} 11.9(2 \mathrm{H}, \mathrm{s}), 7.8(2 \mathrm{H}, \mathrm{t}), 7.7(2 \mathrm{H}, \mathrm{d}), 7.5$ $(2 \mathrm{H}, \mathrm{d}) .{ }^{*}$ Integration normalized to this peak.

SI-20

Figure S8 Solution phase optimized equilibrium structures (no imaginary normal models) of keto isomers of dithranol with explicit solvents calculated at MN15/6-311++G**/SMD level of theory.

SI-21

Figure S9 Gas phase optimized equilibrium structures calculated at MN15/6-311++G** level of theory.

SI-22

Figure S10 Solution phase optimized equilibrium structures (no imaginary normal models) of enol isomers of dithranol with explicit solvents calculated at MN15/6-311++G**/SMD level of theory.

SI-23

Figure S11 Solution phase optimized stationary structures of transient enolate isomers with protonated solvent calculated at MN15/6-311++G**/SMD level of theory. The frozen

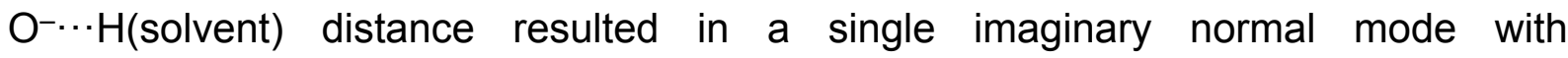
displacements along the $\mathrm{O}^{-\cdots} \mathrm{H}^{+}-\mathrm{S}$ vector.

SI-24

Figure S12 Solution phase optimized equilibrium structures (no imaginary normal models) of enolate form of dithranol with protonated solvent calculated at MN15/6$311++G^{* *} / S M D$ level of theory.

SI-25 
Table S1. Summary of solvent/amino acid side chain correspondence and solvent parameters used from Universal Solvation Model Based on Solute Electron Density (SMD model $\left.{ }^{1}\right) .^{\ddagger}$

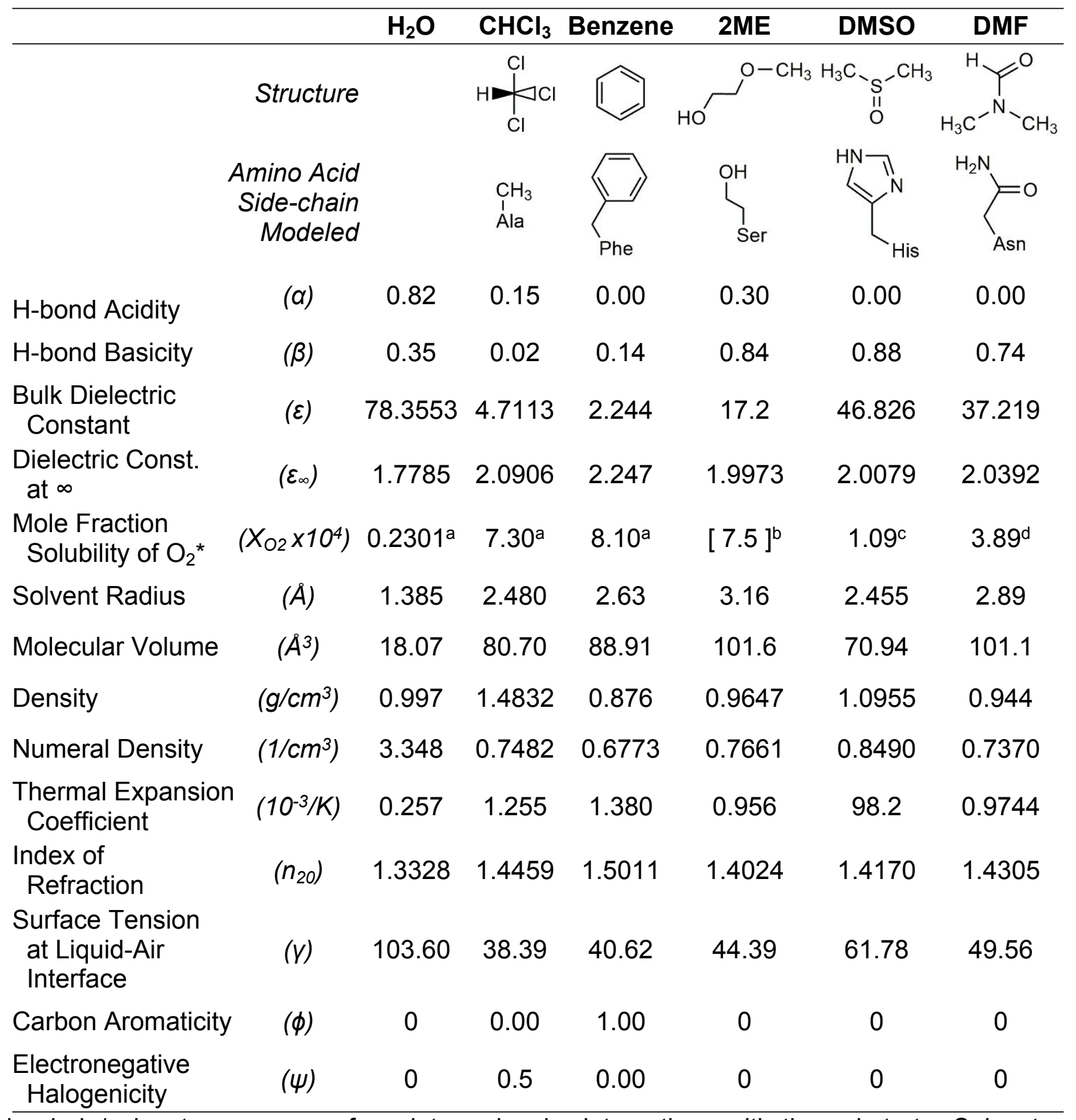

¥ Side chain/solvent groups may form intermolecular interactions with the substrate. Solvents used here to capture the essential chemical properties of diverse amino acid side chains include: $\mathrm{CHCl}_{3}$ (similar to alanine and comparable with a gas phase model), benzene (phenylalanine), DMSO (histidine), DMF (asparagine, glutamine), and $2 \mathrm{ME}$ (serine). Neat $\mathrm{H}_{2} \mathrm{O}$ was used as a control for the no-enzyme condition.

${ }^{*}$ Mole fraction $\left(\mathrm{X}_{\mathrm{O}_{2}}\right)$ of dissolved oxygen at $\mathrm{T}=298 \mathrm{~K}$, partial pressure $\left(\mathrm{O}_{2}\right)=1 \mathrm{~atm}$.
a) Ref. 5
b) Estimate from average of values for diethylether (14.32, Ref. 6) and ethylene glycol (0.723, Ref. 7$)$
c) Ref. 6
d) Ref. 7 
Table S2. Errors in tautomerisation enthalpies $\left(\Delta \Delta^{\mathrm{t}} \mathrm{H}, \mathrm{kJ}^{\mathrm{mol}}{ }^{-1}\right)$ calculated using various density functionals relative to the corresponding CBS-QB3 reference values ${ }^{2}$ for equilibrium structures of gas phase isomers for 1,8-dihydroxyanthrone (keto) and 1,8,9-trihydroxy-anthracane (enol) molecules. ${ }^{\ddagger}$

\begin{tabular}{|c|c|c|c|c|c|}
\hline$\Delta \Delta^{\mathrm{t}} \mathrm{H}, \mathrm{kJ} \mathrm{mol}^{-1}$ & 1HB-keto & 2HB-enol & 1HB-enol & OHB-keto & RMS \\
\hline \multicolumn{6}{|l|}{ Rung 2} \\
\hline BP86 & +8 & +13 & +14 & +21 & +15.0 \\
\hline BLYP & +5 & +10 & +10 & +13 & +9.8 \\
\hline PBE & +7 & +13 & +13 & +20 & +14.2 \\
\hline \multicolumn{6}{|l|}{ Rung 3} \\
\hline TPSS & +7 & +16 & +17 & +19 & +15.5 \\
\hline revTPSS & +6 & +20 & +20 & +16 & +16.3 \\
\hline M06L & +4 & +16 & +13 & +11 & +11.6 \\
\hline \multicolumn{6}{|l|}{ Rung 4} \\
\hline B3LYP & +5 & +6 & +9 & +11 & +8.2 \\
\hline cam-B3LYP & +7 & +13 & +13 & +20 & +14.2 \\
\hline cam-B3LYP+GB3J & +6 & +14 & +17 & +13 & +13.1 \\
\hline$\omega \mathrm{B} 97 \mathrm{XD}$ & +4 & +19 & +20 & +7 & +14.3 \\
\hline MN15 & +3 & +1 & +2 & +7 & +4.0 \\
\hline
\end{tabular}

士The CBS-QB3 tautomerisation enthalpies $\left(\Delta^{t} \mathrm{H}\right)$ relative to $2 \mathrm{HB}$-keto form are taken from Ref 2 as $1 \mathrm{HB}-k$ eto $+47 \mathrm{~kJ} \mathrm{~mol}^{-1}$; $2 \mathrm{HB}$-enol $+52 \mathrm{~kJ} \mathrm{~mol}^{-1}$; 1 $\mathrm{HB}$-enol $+84 \mathrm{~kJ} \mathrm{~mol}^{-1}$; 0HB-keto $+47 \mathrm{~kJ}$ $\mathrm{mol}^{-1}$. All calculations find the 2HB-10-CH2-9-keto form (Scheme S1) to be the most stable isomer. The lowest root-mean-square (RMS) deviation was used to select the density functional with the highest chemical accuracy. The $6-311++G^{* *}$ basis set was used in all calculations. 
Table S3. Summary of nuclear repulsion ( $E_{\text {nuc }}$, a.u. $)$, total electronic energy $\left(E_{S C F}, a . u.\right)$, enthalpy $(H$, a.u.), uncorrected ( $G$, a.u.) and corrected ( $G^{c o r r}$, a.u.) Gibbs free energy, condensed phase translational entropy $\left(\mathrm{J} \mathrm{mol}-1 \mathrm{~K}^{-1}\right.$, at $100 \mu \mathrm{M}$ concentration, $\left.\mathrm{T}=298 \mathrm{~K}\right)$ calculated at $\mathrm{MN} 15 / 6-$ $311++G^{* *} / S M D$ (solvent) level of theory.

\begin{tabular}{|c|c|c|c|c|c|c|}
\hline phase/isomer a & $E_{\text {nuc }}$ & $\mathrm{E}_{\mathrm{SCF}}$ & H & G & $\mathbf{S}^{\text {trans }}$ & Gcor \\
\hline \multicolumn{7}{|l|}{ gas phase } \\
\hline keto & 1142.01524 & -764.53700 & -764.31532 & -764.36776 & 176.2 & -764.3678 \\
\hline enol & 1140.63011 & -764.51652 & -764.29519 & -764.34839 & 176.2 & -764.3484 \\
\hline enolate ${ }^{-}$ & 1126.15900 & -764.01145 & -763.80452 & -763.85538 & 176.1 & -763.8554 \\
\hline \multicolumn{7}{|l|}{ chloroform $\left(\mathrm{CHCl}_{3}\right)$} \\
\hline keto $\times \mathbf{S}$ & 2155.55557 & -2183.61862 & -2183.37015 & -2183.44084 & 190.9 & -2183.4398 \\
\hline enol $\times \mathbf{S}$ & 2056.54026 & -2183.60034 & -2183.35193 & -2183.42503 & 190.9 & -2183.4240 \\
\hline \multicolumn{7}{|l|}{ benzene $\left(\mathrm{C}_{6} \mathrm{H}_{6}\right)$} \\
\hline keto $\times \mathbf{S}$ & 1884.93015 & -996.57625 & -996.24713 & -996.31587 & 188.5 & -996.3149 \\
\hline enol $\times \mathbf{S}$ & 1759.79442 & -996.55467 & -996.22536 & -996.29705 & 188.5 & -996.2829 \\
\hline \multicolumn{7}{|l|}{ Water $\left(\mathrm{H}_{2} \mathrm{O}\right)$} \\
\hline keto $\times \mathbf{S}$ & 1286.15937 & -840.94298 & -840.69559 & -840.75983 & 193.1 & -840.7580 \\
\hline enol $\times \mathbf{S}$ & 1263.76578 & -840.93290 & -840.68562 & -840.74653 & 193.1 & -840.7447 \\
\hline [enolate $\left.^{-} \times \mathrm{SH}^{+}\right]^{*}$ & 1265.04536 & -840.90436 & -840.65720 & -840.71700 & 193.1 & -840.7152 \\
\hline enolate $^{-} \times \mathrm{SH}^{+}$ & 1257.43611 & -840.89258 & -840.64470 & -840.70529 & 193.0 & -840.7035 \\
\hline \multicolumn{7}{|c|}{ 2-methoxyethanol (2ME) } \\
\hline keto $\times \mathbf{S}$ & 1879.21619 & -1033.90527 & -1033.56108 & -1033.63275 & 189.4 & -1033.6317 \\
\hline enol $\times \mathbf{S}$ & 1754.07878 & -1033.88844 & -1033.54468 & -1033.61890 & 189.4 & -1033.6178 \\
\hline$\left[\text { enolate }-\times \mathrm{SH}^{+}\right]^{*}$ & 1768.82969 & -1033.86105 & -1033.51973 & -1033.58759 & 189.4 & -1033.5865 \\
\hline enolate $^{-} \times \mathrm{SH}^{+}$ & 1894.72000 & -1033.43953 & -1033.10918 & -1033.17729 & 189.3 & -1033.1762 \\
\hline \multicolumn{7}{|c|}{ dimethylsulfoxide (DMSO) } \\
\hline keto $\times \mathbf{S}$ & 1888.67638 & -1317.54228 & -1317.23278 & -1317.30249 & 190.2 & -1317.3013 \\
\hline enol $\times \mathbf{S}$ & 1714.39712 & -1317.53408 & -1317.22581 & -1317.29472 & 190.2 & -1317.2935 \\
\hline$\left[\text { enolate }{ }^{-} \times \mathrm{SH}^{+}\right]^{*}$ & 1728.52540 & -1317.52080 & -1317.21524 & -1317.28163 & 190.2 & -1317.2805 \\
\hline enolate ${ }^{-} \times \mathrm{SH}^{+}$ & 1883.98253 & -1317.51733 & -1317.20859 & -1317.27656 & 190.1 & -1317.2754 \\
\hline \multicolumn{7}{|c|}{ N,N-dimethylformamide (DMF) } \\
\hline keto $\times \mathbf{S}$ & 1898.73921 & -1012.86871 & -1012.53578 & -1012.60525 & 189.5 & -1012.6041 \\
\hline enol $\times \mathbf{S}$ & 1704.15983 & -1012.85236 & -1012.51945 & -1012.59302 & 189.5 & -1012.5919 \\
\hline$\left[\text { enolate }-\times \mathrm{SH}^{+}\right]^{*}$ & 1738.61714 & -1012.83713 & -1012.52618 & -1012.50715 & 189.5 & -1012.5710 \\
\hline enolate ${ }^{-} \times \mathbf{S H}^{+}$ & 1840.07851 & -1012.39642 & -1012.07764 & -1012.14720 & 189.4 & -1012.1461 \\
\hline
\end{tabular}

${ }^{a}[]^{\star}$ transient state $\mathrm{w} /$ frozen $\mathrm{O}^{-\cdots} \mathrm{H}^{+}-\mathrm{S}$ distances. 
Table S4. Worksheet for correcting gas phase translational entropy (according to Whitesides et al. ${ }^{3}$ ) to consider condensed phase free volume of in $100 \mu \mathrm{M}$ solution of dithranol and its isomers in the evaluated solvents.

\begin{tabular}{|c|c|c|c|c|c|c|}
\hline & $\mathrm{CHCl} 3$ & $\mathrm{C} 6 \mathrm{H} 6$ & $\mathrm{H} 2 \mathrm{O}$ & 2ME & DMSO & DMF \\
\hline density, g/cm3 & 1.492 & 0.876 & 1.000 & 0.965 & 1.100 & 0.944 \\
\hline [solvent], M & 12.5 & 11.2 & 55.5 & 12.7 & 14.1 & 12.9 \\
\hline numeral density rel. to ideal gas & 2.801E-06 & 2.514E-06 & $1.244 \mathrm{E}-05$ & 2.842E-06 & 3.156E-06 & $2.895 \mathrm{E}-06$ \\
\hline $1 \mathrm{~L}$ of solvent occupies, $\mathrm{mL}$ & 7.5 & 6.8 & 33.4 & 7.6 & 8.5 & $7.8 \mathrm{~V}($ free $)$ macro \\
\hline V available for a solvent, $\AA^{\wedge} 3$ & 132.9 & 148.1 & 29.9 & 131.0 & 117.9 & 128.6 \\
\hline Inter Solvent Distance, $\AA$ & 5.10 & 5.29 & 3.10 & 5.08 & 4.90 & 5.05 \\
\hline V of a molecule, $\AA^{\wedge} 3$ & 104 & 107 & 25 & 102 & 96 & 101 \\
\hline solvent radius, $\AA$ & 2.920 & 2.945 & 1.814 & 2.895 & 2.840 & 2.889 \\
\hline MOPAC PM7 COSMO V, $\AA^{\wedge} 3$ & 104 & 107 & 25 & 101 & 96 & 101 \\
\hline MOPAC PM7 COSMO A, Å^2 & 116 & 119 & 42 & 117 & 110 & 115 \\
\hline G16PCM cavity $\mathrm{V}, \AA^{\wedge} 3$ & 141 & 134 & 19 & 111 & 111 & 125 \\
\hline G16PCM cavity A, $\AA^{\wedge} 2$ & 145 & 143 & 36 & 124 & 124 & 138 \\
\hline SMD Rsolv, $\AA$ & 2.48 & 2.63 & 1.39 & 2.46 & 2.46 & \\
\hline SMD Vmmol, $\AA^{\wedge} 3$ & 80.70 & 88.91 & 18.07 & 70.94 & 70.94 & \\
\hline $\mathrm{E}$ (solvent molecule), $\AA$ & 4.71 & 4.75 & 2.92 & 4.67 & 4.58 & 4.66 \\
\hline $\mathrm{V}$ (non-excluded void), $\AA^{\wedge} 3$ & 29 & 41 & 5 & 29 & 22 & 28 \\
\hline$E($ free $), \AA$ & 0.73 & 1.09 & 0.33 & 0.82 & 0.60 & 0.72 \\
\hline $\mathrm{V}($ free $)$ for single, $\AA^{\wedge} 3$ & 0.39 & 1.28 & 0.04 & 0.56 & 0.22 & $0.37 \mathrm{~V}$ (free) nano \\
\hline shape: $8=$ cube; 6.3 sphere & 6.3 & 8 & 6.3 & 8 & 6.3 & 6.3 \\
\hline Mw (solvent), g/mol & 119.4 & 78.1 & 18.0 & 76.1 & 78.1 & 73.1 \\
\hline Gas phase & \multicolumn{6}{|c|}{$36.9+12.5 \ln M+12.5 \ln T$} \\
\hline standard state, $[\mathrm{X}] \mathrm{mol} / \mathrm{L}$ & 0.0446 & 0.0446 & 0.0446 & 0.0446 & 0.0446 & $0.04461 \mathrm{~mol}=22.4 \mathrm{~L}$ \\
\hline approx. Strans, J/molK & 168.0 & 162.7 & 144.3 & 162.3 & 162.7 & 161.8 \\
\hline part 1, Eq.2 & -233.2 & -233.2 & -233.2 & -233.2 & -233.2 & -233.2 \\
\hline part 2, Eq.2 & 253.3 & 252.7 & 250.5 & 252.7 & 252.7 & 252.6 \\
\hline exact calc. Strans & 167.7 & 162.4 & 144.1 & 162.0 & 162.4 & $161.5 \mathrm{~J} / \mathrm{mol} \mathrm{K}$ \\
\hline & 40.1 & 38.8 & 34.5 & 38.8 & 38.8 & $38.6 \mathrm{cal} / \mathrm{mol} \mathrm{K}$ \\
\hline pure liquid phase & \multicolumn{6}{|c|}{$-8.0+12.5 \ln M+12.5 \ln T$} \\
\hline$[\mathrm{X}] \mathrm{mol} / \mathrm{L}$ & 12.5 & 11.2 & 55.5 & 12.7 & 14.1 & 12.9 std state $\sim 10 \mathrm{M}$ \\
\hline approx. Strans, J/molK & 121.1 & 116.7 & 85.1 & 115.4 & 114.8 & 114.7 diff in. $[X]$ not - \\
\hline part 1, Eq.2 & -238.8 & -238.7 & -240.3 & -238.8 & -238.9 & -238.8 \\
\hline exact calc. Strans & 120.8 & 116.4 & 84.8 & 115.1 & 114.5 & 114.4 \\
\hline condensation entropy, $\mathrm{J} / \mathrm{molK}$ & -46.9 & -46.0 & -59.3 & -47.0 & -47.8 & -47.1 exp: $\sim-88 \mathrm{~J} / \mathrm{moll}$ \\
\hline
\end{tabular}

Improved liquid phase [X] mol/L corr w/B20 or /B23 approx. Strans, J/molK part 1, Eq.2 exact calc. Strans

condensation entropy, $\mathrm{J} / \mathrm{molK}$

Solutions [analyte], $\mathrm{mmol} / \mathrm{L}$ Mw (analyte), g/mol effect. [analyte] in solvent, $\mathrm{mol} / \mathrm{L}$

$$
\begin{array}{r}
\text { simple model } \\
\text { approx. Strans } \\
\text { using the eff. [analyte] or Vfree } \\
\text { approx. Strans } \\
\text { change } \\
\\
\\
\text { approx. Strans, J/molK } \\
\text { part } 1, \text { Eq.2 } \\
\text { part } 2 \text { w/analyte Mw, Eq.2 } \\
\text { exact calc. Strans }
\end{array}
$$

\section{4 \\ 121.1 \\ $-244.6$ \\ 72.3}

$-95.3$

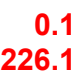

226.1

0.013

\section{solu}

solute trans. S dominated by SOLVENT molecules

solvent more conc. w/respect to translational S

$\begin{array}{rrrrr}1297 & 44988 & 2964 & 7664 & 4429 \text { non-excl vs. free V } \\ 116.7 & 85.1 & 115.4 & 114.8 & 114.7 \\ -243.5 & -247.0 & -244.3 & -245.2 & -244.7 \\ 76.9 & 29.1 & 69.7 & 62.2 & 65.9 \\ -85.5 & -114.9 & -92.3 & -100.2 & -95.7 \text { exp: } \sim-88 \mathrm{~J} / \mathrm{molK}\end{array}$

\begin{tabular}{|c|c|c|c|c|c|}
\hline 227 & 227 & 227 & 227 & 227 & 227 \\
\hline & $1.1+12.5 !$ & $12.5 \ln T-$ & Tanalyte, & ive] & \\
\hline 186 & 185 & 198 & 186 & 187 & 186 \\
\hline $82 \%$ & $82 \%$ & $88 \%$ & $82 \%$ & $83 \%$ & $82 \%$ \\
\hline 186.0 & 185.1 & 198.4 & 186.1 & 187.0 & 186.3 \\
\hline-232.0 & -232.1 & -230.5 & -232.0 & -231.8 & -231.9 \\
\hline 254.3 & 254.3 & 254.3 & 254.3 & 254.3 & 254.3 \\
\hline 185.7 & 184.8 & 198.1 & 185.8 & 186.7 & 186. \\
\hline
\end{tabular}

$\begin{array}{rrrr}0.1 & 0.1 & 0.1 & 0.1 \\ 226.1 & 226.1 & 226.1 & 226.1 \\ 0.015 & 0.003 & 0.013 & 0.012\end{array}$

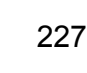

0.015

0.003

0.013

0.012

226.1 dithranol

$0.013 \mathrm{~V}$ free is for solvent 
Table S5. Summary of UV/visible absorbance data for dithranol and the oxygenation product dithranone: Wavelengths $(\mathrm{nm})$ of peak maxima and shoulders.

\begin{tabular}{|c|c|c|c|c|c|c|c|c|c|c|}
\hline [Dithranol] & Solvent & $\lambda_{1}$ & $\lambda_{2}$ & $\lambda_{3}$ & $\lambda_{4}$ & $\lambda_{5}$ & $\lambda_{6}$ & $\lambda_{7}$ & $\lambda_{8}$ & $\lambda_{9}$ \\
\hline $50 \mu \mathrm{M}$ & $\mathrm{CHCl}_{3}$ & $258^{b}$ & 288 & - & $358^{a}$ & - & - & - & - & - \\
\hline $50 \mu \mathrm{M}$ & benzene & $-d$ & 289 & - & $359^{a}$ & - & - & - & - & - \\
\hline $50 \mu \mathrm{M}$ & DMSO & $265^{b}$ & 289 & - & 358 & 372 & $393^{a}$ & 415 & 453 & 480 \\
\hline $50 \mu \mathrm{M}$ & $\mathrm{DMSO}_{\text {Acid }}$ & $261^{d}$ & 291 & - & 357 & - & - & - & - & - \\
\hline $50 \mu \mathrm{M}$ & $\mathrm{DMSO}_{\text {Alk }}$ & 269 & $289^{c}$ & 312 & $357^{c}$ & 374 & $394^{a}$ & 429 & 454 & 480 \\
\hline $50 \mu \mathrm{M}$ & $2 \mathrm{ME}$ & $263^{b}$ & 289 & - & 358 & $367^{a}$ & 389 & 424 & 443 & 475 \\
\hline $50 \mu \mathrm{M}$ & $2 \mathrm{ME}_{\text {Acid }}$ & $-d$ & 290 & - & $358^{a}$ & - & - & - & - & - \\
\hline $50 \mu \mathrm{M}$ & $2 \mathrm{ME}_{\mathrm{Alk}}$ & $-d$ & 289 & $310^{c}$ & $354^{c}$ & 371 & $390^{a}$ & 425 & 449 & 470 \\
\hline $50 \mu \mathrm{M}$ & DMF & $-d$ & $-d$ & 311 & $355^{c}$ & 373 & $392^{a}$ & 428 & 453 & 479 \\
\hline $25 \mu \mathrm{M}$ & $\mathrm{H}_{2} \mathrm{O}$ & $258^{b}$ & 286 & - & $351^{a}$ & $389^{c}$ & - & - & - & - \\
\hline [Dithranone] & Solvent & $\lambda_{1}$ & $\lambda_{2}$ & $\lambda_{3}$ & $\lambda_{4}$ & $\lambda_{5}$ & $\lambda_{6}$ & $\lambda_{7}$ & $\lambda_{8}$ & $\lambda_{9}$ \\
\hline $50 \mu \mathrm{M}$ & $\mathrm{CHCl}_{3}$ & $248^{b}$ & 286 & - & $432^{a}$ & - & - & - & - & - \\
\hline $50 \mu \mathrm{M}$ & benzene & $-^{d}$ & 281 & - & $432^{a}$ & - & - & - & - & - \\
\hline $50 \mu \mathrm{M}$ & DMSO & $266^{b}$ & $283^{c}$ & - & $428^{a}$ & - & - & - & - & - \\
\hline $50 \mu \mathrm{M}$ & $\mathrm{DMSO}_{\text {Acid }}$ & $266^{b}$ & $283^{c}$ & - & $429^{a}$ & - & - & - & - & - \\
\hline $50 \mu \mathrm{M}$ & $\mathrm{DMSO}_{\mathrm{Alk}}$ & $-d$ & 284 & 332 & - & $565^{a}$ & - & - & - & - \\
\hline $50 \mu \mathrm{M}$ & $2 \mathrm{ME}$ & $-d$ & $283^{b}$ & - & 432 & $520^{a}$ & - & - & - & - \\
\hline $50 \mu \mathrm{M}$ & $2 \mathrm{ME}_{\text {Acid }}$ & $-d$ & 284 & - & - & - & - & - & - & - \\
\hline $50 \mu \mathrm{M}$ & $2 \mathrm{ME}_{\mathrm{Alk}}$ & $-d$ & 284 & $316^{c}$ & - & $517^{a}$ & - & - & - & - \\
\hline $50 \mu \mathrm{M}$ & DMF & 274 & 284 & - & $430^{b}$ & - & - & - & - & - \\
\hline $25 \mu \mathrm{M}$ & $\mathrm{H}_{2} \mathrm{O}$ & $273^{b}$ & $287^{c}$ & - & $412^{a}$ & $470^{c}$ & - & - & - & - \\
\hline
\end{tabular}
a) Visible absorbance maximum (>300 $\mathrm{nm}$ )
b) Absorbance maximum (depends on UV-transparency of solvent)
c) Shoulder; "peak" wavelength is estimated
d) Not measured 
Table S6. Summary of fluorescence data for dithranol: Wavelengths $(\mathrm{nm})$ of peak maxima and shoulders. The upper section summarizes dithranol fluorescence emission spectra while the lower section summarizes fluorescence excitation spectra, where light emission at $495 \mathrm{~nm}$ was monitored. Note: The oxygenation product, dithranone, does not fluoresce under any conditions tested.

\begin{tabular}{|c|c|c|c|c|c|c|c|c|c|c|c|c|}
\hline Conc. & Solvent & $\lambda_{\mathrm{Exc}}$ & $\lambda_{1}$ & $\lambda_{2}$ & $\lambda_{3}$ & $\lambda_{4}$ & $\lambda_{5}$ & & & & & \\
\hline $50 \mu \mathrm{M}$ & $\mathrm{CHCl}_{3}$ & 380 & 454 & $481^{a}$ & - & 591 & - & & & & & \\
\hline $50 \mu \mathrm{M}$ & Benzene & 380 & 465 & $493^{a}$ & $530^{c}$ & $585^{c}$ & - & & & & & \\
\hline $5 \mu \mathrm{M}$ & DMSO & 393 & - & 499 & $531^{a}$ & 565 & $617^{c}$ & & & & & \\
\hline $5 \mu \mathrm{M}$ & $\mathrm{DMSO}_{\text {Acidic }}$ & 392 & - & $499^{a}$ & 534 & $566^{c}$ & $617^{c}$ & & & & & \\
\hline $5 \mu \mathrm{M}$ & $\mathrm{DMSO}_{\mathrm{Alk}}$ & 392 & - & 498 & $531^{a}$ & 565 & $617^{c}$ & & & & & \\
\hline $5 \mu \mathrm{M}$ & $2 \mathrm{ME}$ & 389 & - & 495 & $528^{a}$ & 565 & $613^{c}$ & & & & & \\
\hline $5 \mu \mathrm{M}$ & $2 \mathrm{ME}_{\text {Acidic }}$ & 388 & $459^{c}$ & $491^{a}$ & $524^{c}$ & $570^{c}$ & - & & & & & \\
\hline $5 \mu \mathrm{M}$ & $2 \mathrm{ME}_{\mathrm{Alk}}$ & 389 & - & 495 & $528^{a}$ & $565^{c}$ & $613^{c}$ & & & & & \\
\hline $5 \mu \mathrm{M}$ & DMF & 391 & - & 496 & $530^{a}$ & 564 & $617^{c}$ & & & & & \\
\hline $25 \mu \mathrm{M}$ & $\mathrm{H}_{2} \mathrm{O}$ & 383 & - & 492 & $524^{a}$ & $560^{c}$ & $611^{c}$ & & & & & \\
\hline Conc. & Solvent & $\lambda_{\mathrm{Em}}$ & $\lambda_{1}$ & $\lambda_{2}$ & $\lambda_{3}$ & $\lambda_{4}$ & $\lambda_{5}$ & $\lambda_{6}$ & $\lambda_{7}$ & $\lambda_{8}$ & $\lambda_{9}$ & $\lambda_{10}$ \\
\hline $50 \mu \mathrm{M}$ & $\mathrm{CHCl}_{3}$ & 495 & $248^{c}$ & $262^{b}$ & - & - & $364^{c}$ & $380^{a}$ & 410 & 430 & - & - \\
\hline $5 \mu \mathrm{M}$ & Benzene & 495 & $-^{d}$ & $-d$ & - & - & 363 & $380^{a}$ & - & 429 & - & - \\
\hline $5 \mu \mathrm{M}$ & DMSO & 495 & - & 267 & - & $354^{c}$ & 373 & $393^{b}$ & - & 427 & 453 & 480 \\
\hline $5 \mu \mathrm{M}$ & $\mathrm{DMSO}_{\text {Acidic }}$ & 495 & - & 268 & - & - & $380^{c}$ & $392^{b}$ & - & 429 & 451 & 480 \\
\hline $5 \mu \mathrm{M}$ & $\mathrm{DMSO}_{\mathrm{Alk}}$ & 495 & - & 268 & 313 & $354^{c}$ & 373 & $392^{b}$ & 417 & 428 & 453 & 480 \\
\hline $5 \mu \mathrm{M}$ & 2ME & 495 & - & $264 b$ & - & $354^{c}$ & 370 & $389^{a}$ & - & 425 & 447 & $479^{\circ}$ \\
\hline $5 \mu \mathrm{M}$ & $2 \mathrm{ME}_{\text {Acidic }}$ & 495 & - & $305^{\mathrm{a}}$ & - & - & $370^{c}$ & 387 & - & $430^{c}$ & - & - \\
\hline $5 \mu \mathrm{M}$ & $2 \mathrm{ME}_{\mathrm{Alk}}$ & 495 & - & $263 b$ & - & $354^{c}$ & 371 & $389^{a}$ & - & 425 & 447 & 479 \\
\hline $5 \mu \mathrm{M}$ & DMF & 495 & - & 271 & $311^{c}$ & $354^{c}$ & 372 & $392^{b}$ & - & 426 & 450 & - \\
\hline $25 \mu \mathrm{M}$ & $\mathrm{H}_{2} \mathrm{O}$ & 495 & $246^{c}$ & $260 \mathrm{~b}$ & - & $354^{c}$ & 365 & $383^{a}$ & 410 & 426 & 441 & - \\
\hline
\end{tabular}

a) Emission maximum or (visible, $>300 \mathrm{~nm}$ ) excitation maximum

b) Excitation maximum (depends on UV-transparency of solvent)

c) Shoulder; "peak" wavelength is estimated

d) Not measured 
<smiles>O=C1c2c(O)cccc2[CH][C]2c3cccc(O)c3C(=O)C21</smiles>

10- $\mathrm{CH}_{2}$<smiles>O=C1c2c(O)cccc2[CH]c2cccc(O)c21</smiles>

9-keto<smiles></smiles>

2HB

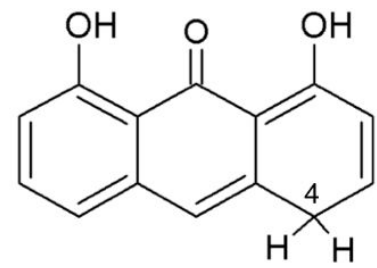

4- $\mathrm{CH}_{2}$<smiles></smiles>

3- $\mathrm{CH}_{2}-\mathrm{zW}$<smiles>O=C1C2=C(O)CC=CC2=Cc2cccc(O)c21</smiles>

2- $-\mathrm{CH}_{2}$<smiles></smiles>

1-keto<smiles>O=C1c2c(O)cccc2[CH][CH][CH]c2cccc(O)c21</smiles>

1HB<smiles>Oc1cccc2cc3cccc(O)c3c(O)c12</smiles>

enol

OHB<smiles>O=c1c2c(O)cccc2[pH]c2cccc(O)c12</smiles>

10-C<smiles></smiles>

4-C<smiles>[O-]c1c2c(O)cccc2cc2cccc(O)c12</smiles>

3-C--zw<smiles></smiles>

2-C

9-enolate<smiles>[O-]c1cccc2cc3cccc(O)c3c(O)c12</smiles>

1-enolate<smiles>O=C1c2c([O-])cccc2[CH]c2cccc(O)c21</smiles>

9-keto, 1-enolate

Scheme S1. Overview of dithranol tautomers with key $\mathrm{C}-\mathrm{H}$ and $\mathrm{O}-\mathrm{H}$ positional variations and representative hydrogen bonding patterns. 

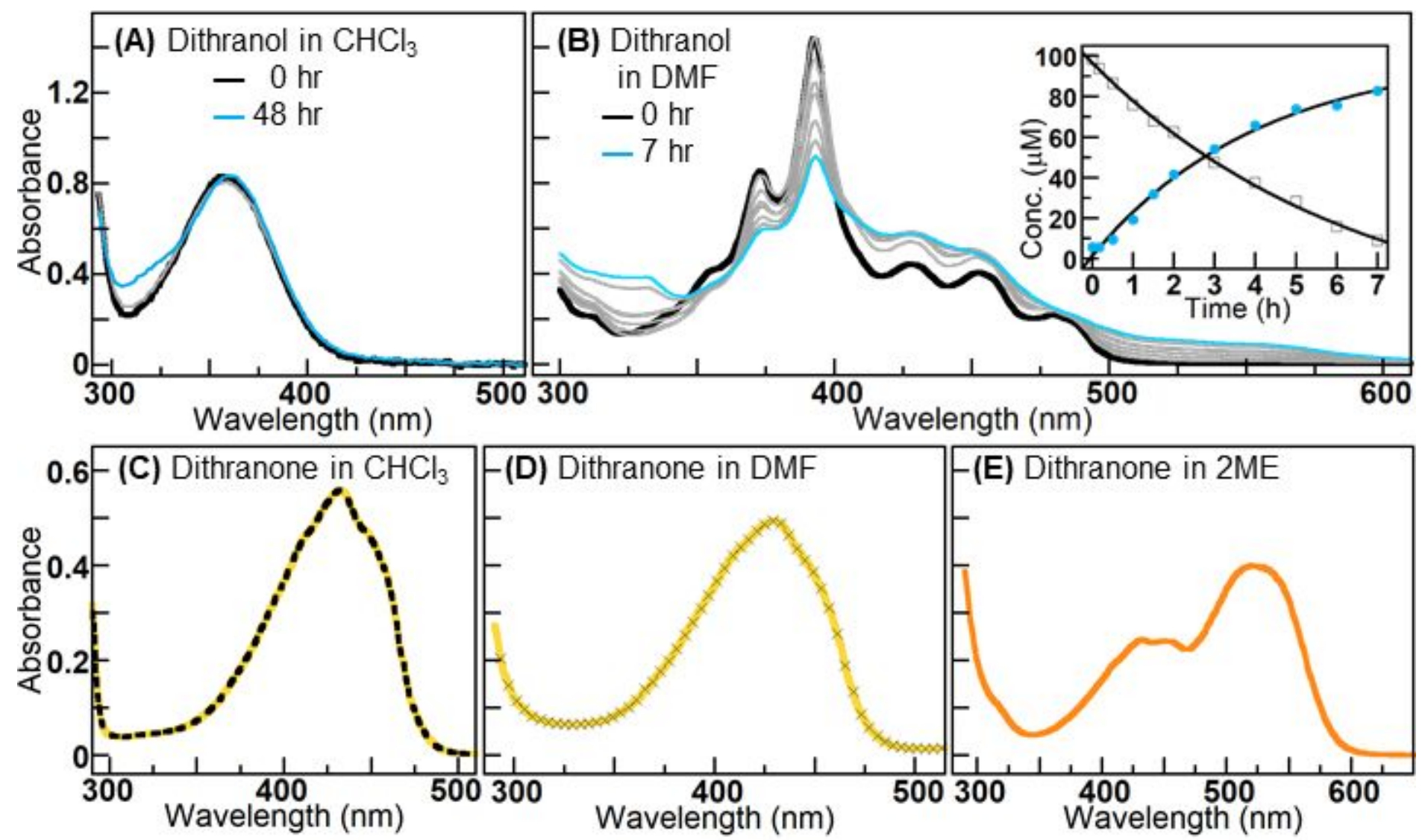

Figure S1. The rate of dithranol oxidation in air varies with solvent, according to the initial fraction of enolate present, as illustrated by time resolved UV/vis spectroscopy and HPLC. Reactions of dithranol in air (room temperature, $100 \mathrm{rpm}$ shaking, air-saturation) were monitored over time by UV/vis absorbance in (A) $\mathrm{CHCl}_{3}(124 \mu \mathrm{M}$ dithranol) and (B) DMF (110 $\mu \mathrm{M}$ dithranol). (B, inset) Discontinuous HPLC was used to monitor the disappearance of dithranol (white squares) and appearance of dithranone (blue circles) over time in DMF. The data were fit to single exponential curves yielding $\mathrm{k}=0.15 \mathrm{~h}$ (dithranol disappearance) or $\mathrm{k}=0.24 \mathrm{~h}$ (dithranone appearance). Dithranol oxidation can occur by $\mathrm{O}_{2}$-dependent pathways that lead either to the dimerization product (bisanthrone) or monooxygenation (dithranone). The least error in HPLC determination of progress of reaction was previously shown to occur by monitoring dithranone production, which has $\mathrm{k}=0.24 \mathrm{~h}$ or $\mathrm{t}_{1 / 2}=2.9 \mathrm{~h}$. (C-E) UV/vis absorbance spectra of the oxygenation product, dithranone (50 $\left.\mu \mathrm{M}\right)$, in (C) $\mathrm{CHCl}_{3}$, (D) DMF, and (E) 2ME. 


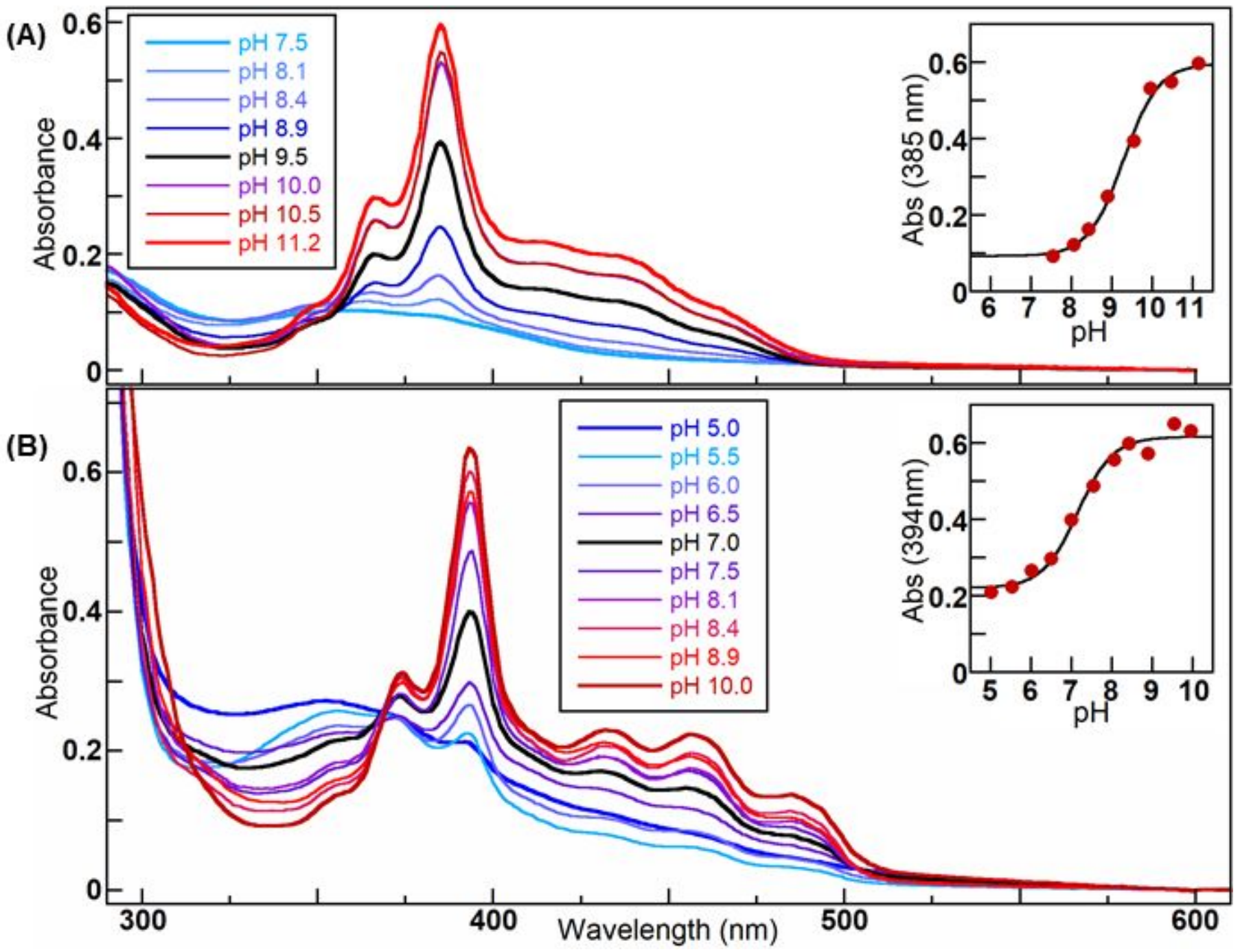

Figure S2. NMO lowers the pKa of dithranol by greater than $2 \mathrm{pH}$ units. Spectrophotometric $\mathrm{pH}$ titrations of (A) $46 \mu \mathrm{M}$ dithranol and (B) $39 \mu \mathrm{M}$ dithranol mixed with $80 \mu \mathrm{M}$ NMO (NMO-dithranol complex) were performed discontinuously using a series of aqueous buffers containing $100 \mathrm{mM}$ buffer and $300 \mathrm{mM} \mathrm{NaCl}$ pre-adjusted to $\mathrm{pH} 5.02,5.53,5.97,6.50,7.00,7.57,8.06,8.43,8.90,9.54$, 9.95, 10.47, 11.15 (see Solvents and Reagents section of Experimental). An approximate isosbestic point at $345 \mathrm{~nm}$ is observed in panel A. An isosbestic point at $368 \mathrm{~nm}$ is observed in panel B. Upon binding to the enzyme, the absorbance maxima in dithranol's high-pH spectrum are observed to red shift by approximately $8 \mathrm{~nm}$. The individual peaks, especially above $400 \mathrm{~nm}$, are more well-resolved. (Insets) $\mathrm{p} K_{a}$ values were determined by plotting the absorbance at $385 \mathrm{~nm}$ (dithranol) or $394 \mathrm{~nm}$ (NMO-dithranol complex) as a function of $\mathrm{pH}$ and fitting the data to a sigmoidal curve. The active site pocket of NMO lowers the $p K_{a}$ of dithranol from $9.28 \pm 0.07$ to $7.15 \pm 0.09\left(\Delta p K_{a}=2.13 \pm 0.11\right)$. 


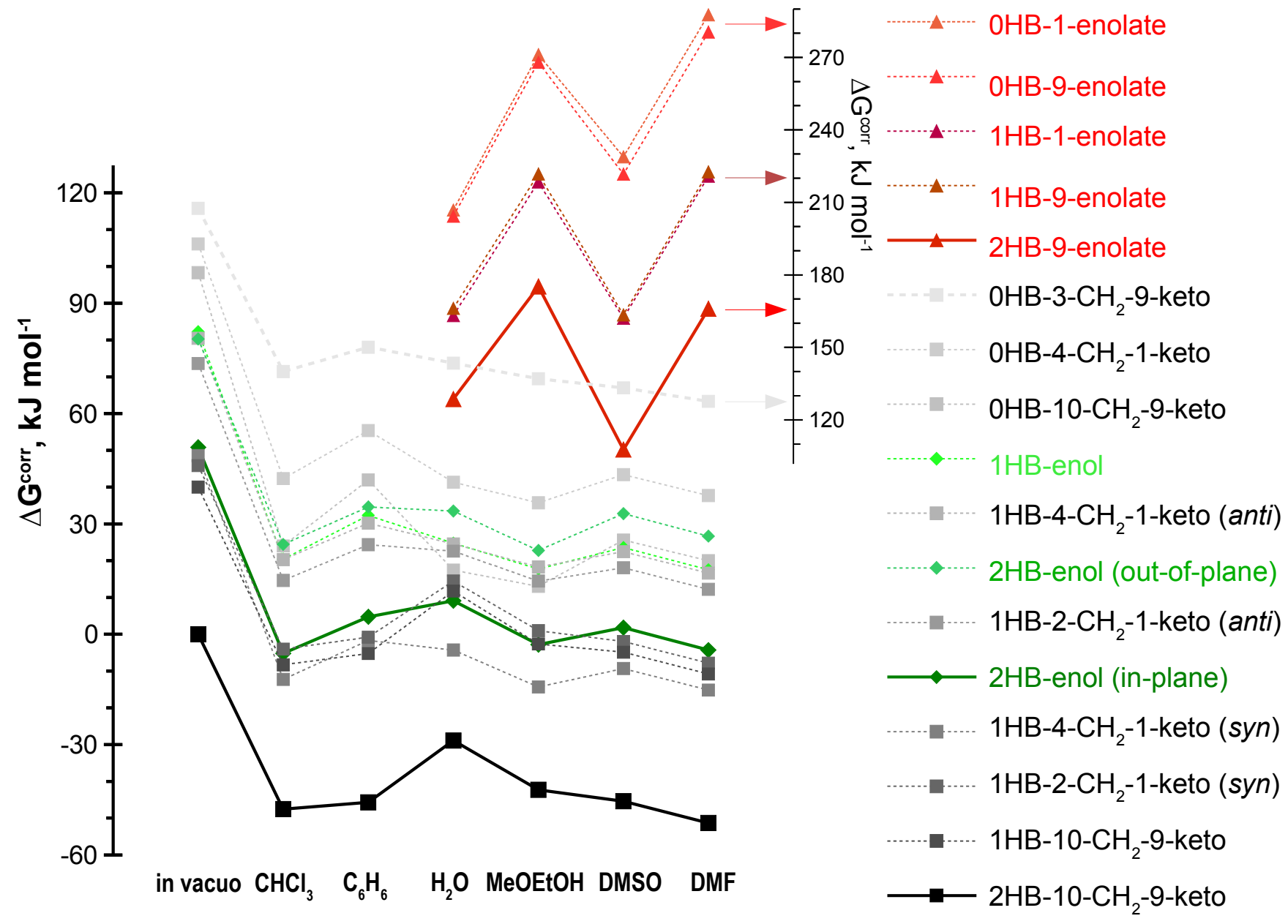

Figure S3. Gibbs free energy landscape (corrected for translational entropy in $100 \mu \mathrm{M}$ solution at MN15/6-311++G**/SMD level of theory) of dithranol tautomers as defined in Scheme S1. All energy values are given relative to the lowest energy keto isomer in gas phase. Isomers are ordered according to their relative energies in gas phase as shown on the left-hand side of the figure. Enolate structures were mapped considering protonated solvents at infinite separation and both embedded in polarizable continuum model SMD. 

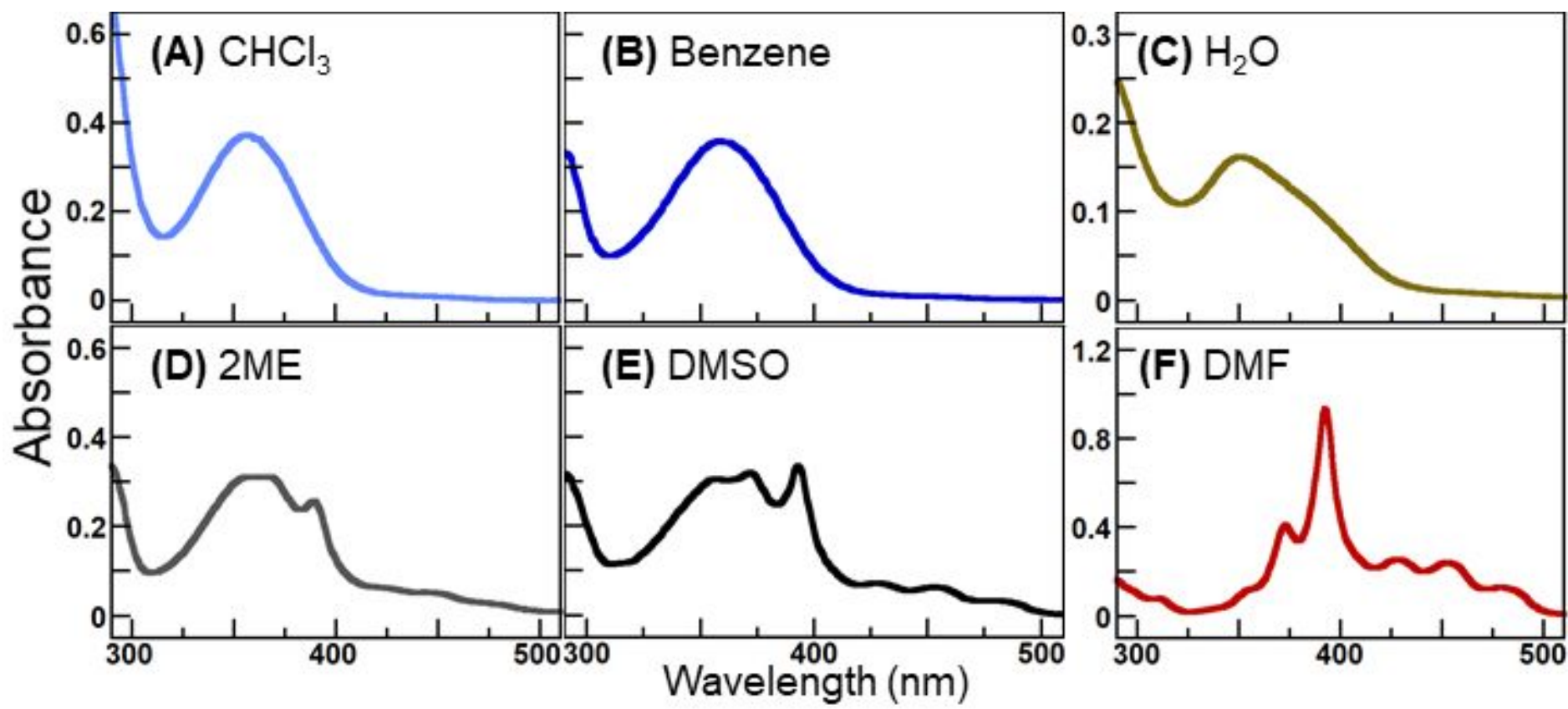

Figure S4. The UV/vis absorbance spectrum of dithranol is solvent-dependent. Representative spectra of $50 \mu \mathrm{M}$ dithranol in (A) $\mathrm{CHCl}_{3}$, (B) benzene, (C) $\mathrm{H}_{2} \mathrm{O}(25 \mu \mathrm{M}$ dithranol) (D) 2ME, (E) DMSO, and (F) DMF. In $\mathrm{CHCl}_{3}$ and benzene, the spectrum of dithranol exhibits a single broad peak centered at 358 and $359 \mathrm{~nm}$, respectively, which is similar to that observed in aqueous buffer at low $\mathrm{pH}(<1$ unit from the $p K_{\mathrm{a}}$ ). (See Figure S2A.) The spectrum in pure distilled water (C) resembles the spectra shown in (A) and (B); however, the source of asymmetry in this spectrum is unclear. In DMF, dithranol's spectrum is similar to the NMO-dithranol spectrum measured in high $\mathrm{pH}\left(>1\right.$ unit from $\mathrm{pK} K_{\mathrm{a}}$ ) aqueous buffer: dominated by a sharp peak at $391 \mathrm{~nm}$ with a shoulder at $373 \mathrm{~nm}$ and three minor peaks at 428, 453, and $479 \mathrm{~nm}$. (See Figure S2B.) The bathochromic shift and appearance of additional peaks at higher wavelengths are indicative of increased conjugation (e.g., see spectra for benzene, naphthalene, and anthracene, representing increases in conjugation) consistent with the assignment of a ketone isomer to the single-peak spectrum and a more-conjugated, enol(ate) isomer to the multi-peak, red-shifted spectrum. In DMSO and 2ME, dithranol's spectrum exhibits features of both $\mathrm{pH}$-extremes, suggesting that dithranol exists as a mixture of the two species in these solvents. (See Figure S5). 


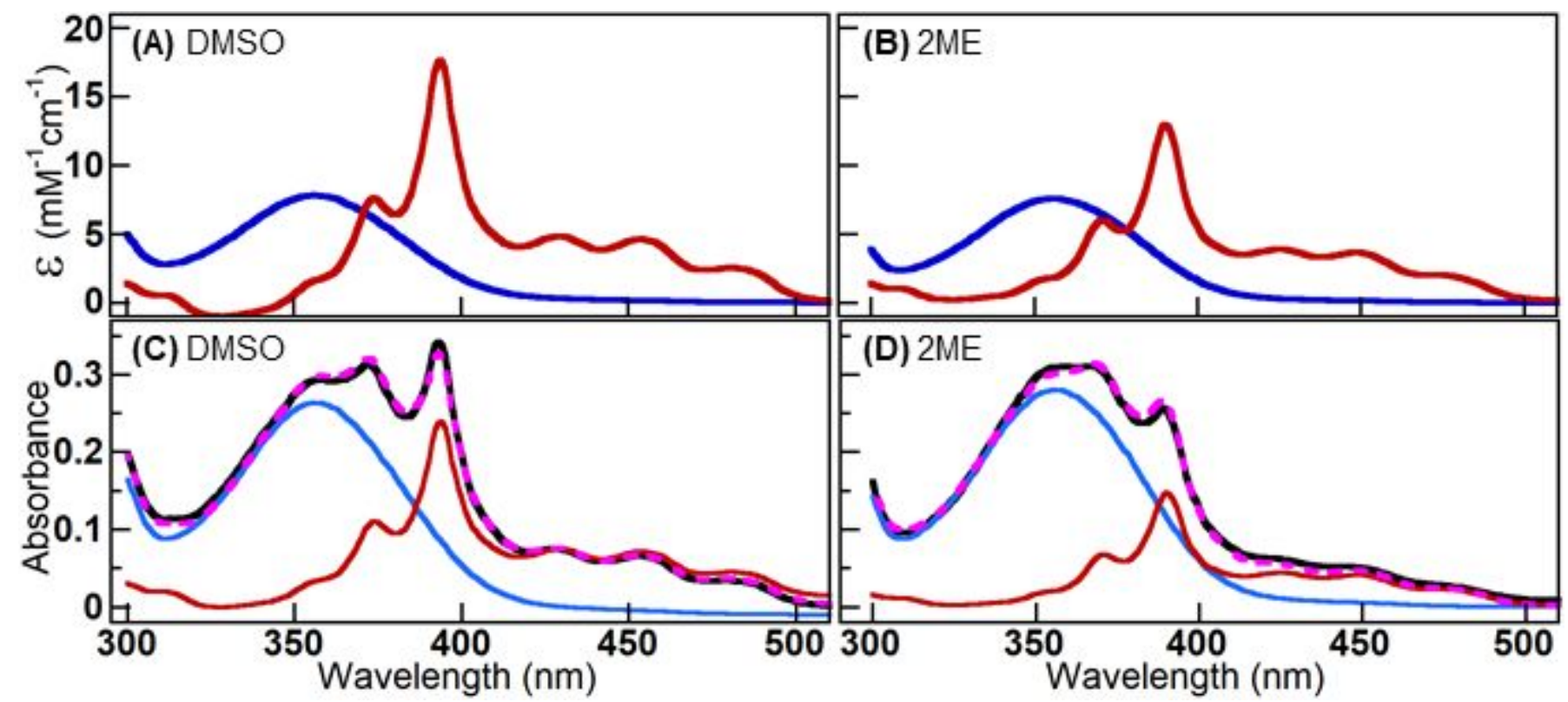

Figure S5. The absorbance spectra of dithranol in neat DMSO or 2 ME can be simulated as linear combinations of spectra measured in acidic or alkaline solvent. UV/Vis absorption spectra of dithranol at varying concentrations were measured in DMSO or 2ME in the presence of excess (1 $\mathrm{mM}) \mathrm{HCl}$ or $\mathrm{NaOH}$. Simulated absorbance spectra were generated as linear combinations of the two component spectra (acidic and alkaline) representing the protonated ketone and deprotonated enolate forms of dithranol. Molar absorptivity is plotted as a function of wavelength for dithranol in (A) $\mathrm{DMSO}_{\text {Acidic }}$ (blue) or $\mathrm{DMSO}_{\text {Alk }}$ (red) and (B) $2 \mathrm{ME}_{\text {Acidic }}$ (blue) or $2 \mathrm{ME}_{\text {Alk }}$ (red). (C) The absorbance spectrum of $50 \mu \mathrm{M}$ dithranol in neat DMSO (black) and a simulated spectrum (pink/dashed) that was generated as the sum of the weighted component spectra shown in blue $(73 \%)$ and red $(27 \%)$. (D) Absorbance spectrum of $50 \mu \mathrm{M}$ dithranol in $2 \mathrm{ME}$ (black) and a simulated spectrum (pink/dashed) that was generated as the sum of the weighted component spectra shown in blue $(76 \%)$ and red $(24 \%)$. 


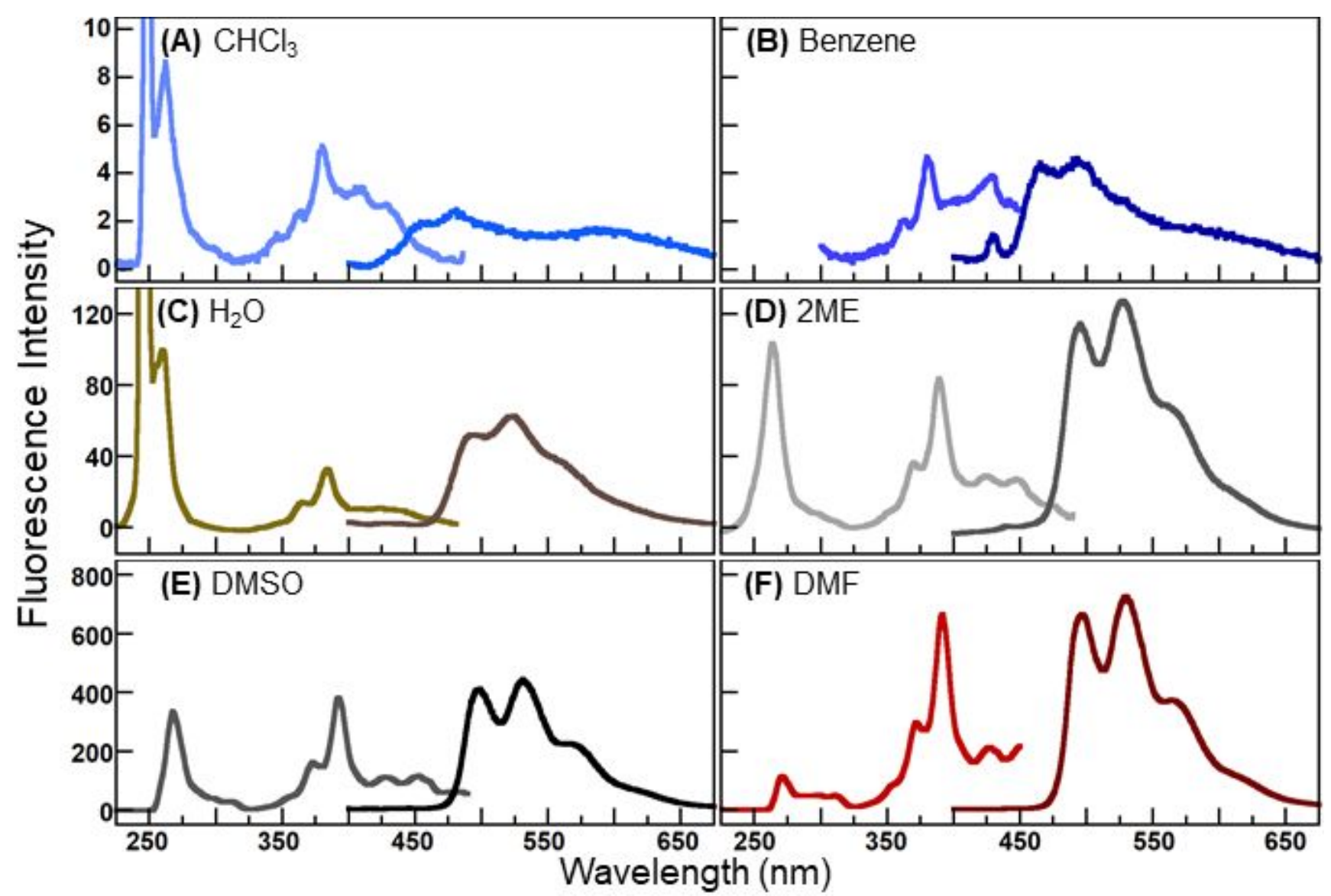

Figure S6. The fluorescence emission intensity and excitation spectra of dithranol show different solvent-dependent trends. Excitation spectra were measured by illuminating dithranol over a range of wavelengths (lighter-colored lines, 250-470 nm) and monitoring emitted fluorescence at $495 \mathrm{~nm}$. The emission spectra (darker lines, 400-650 nm) were then produced by exciting dithranol at the visible wavelength at which maximum fluorescence intensity was observed in the excitation spectrum $\left(\lambda_{\mathrm{Exc}}\right)$. Representative spectra are shown in (A) $\mathrm{CHCl}_{3}\left(50 \mu \mathrm{M}, \lambda_{\mathrm{Exc}}=380 \mathrm{~nm}\right)$, (B) benzene $\left(50 \mu \mathrm{M}, \lambda_{\mathrm{Exc}}=380 \mathrm{~nm}\right),(C) \mathrm{H}_{2} \mathrm{O}\left(25 \mu \mathrm{M}, \lambda_{\mathrm{Exc}}=383 \mathrm{~nm}\right)$, (D) $2 \mathrm{ME}\left(5 \mu \mathrm{M}, \lambda_{\mathrm{Exc}}=389 \mathrm{~nm}\right),(\mathrm{E}) \mathrm{DMSO}$ $\left(5 \mu \mathrm{M}, \lambda_{\mathrm{Exc}}=393 \mathrm{~nm}\right)$, and (F) DMF $\left(5 \mu \mathrm{M}, \lambda_{\mathrm{Exc}}=391 \mathrm{~nm}\right)$. Little to no fluorescence was observed in solutions of dithranol in $\mathrm{CHCl}_{3}$ and benzene. By contrast, dithranol fluoresces orders of magnitude more intensely in DMF than any other neat solvent. (Note the differences in the various y-scales.) Intermediate fluorescence intensity is observed in neat DMSO or $2 \mathrm{ME}$, which can be increased by addition of $\mathrm{NaOH}$ (not shown). Keto forms (Scheme S1) lacking conjugation in the center ring are expected be flexible, diminishing conjugation in the 3-ring system and consequently lowering fluorescence. The intense transitions in dithranol's fluorescence emission spectrum in DMF are consistent with a fully conjugated, planar 3-ring system. The fluorescence spectra of dithranol in DMSO and $2 \mathrm{ME}$ are of intermediate intensity, reflecting the mixed ketone/enol(ate) composition. The small amount of fluorescence observed in solutions of dithranol in $\mathrm{CHCl}_{3}$ and benzene could suggest that a very small fraction of dithranol exists as a planar enol in those solvents. This is supported by the shape of the fluorescence excitation spectra $(200-450 \mathrm{~nm})$ when emission at $495 \mathrm{~nm}$ is monitored. This has a maximum near $380 \mathrm{~nm}$ with small peaks at higher wavelengths, rather than a single broad peak near $360 \mathrm{~nm}$ as in the absorbance spectrum for the ketone, which would be expected if the ketone isomer itself was minimally fluorescent, e.g. while transiently planar. This blueshift in maximum excitation wavelength is accompanied by a blue-shift in the entire emission spectrum, which suggests that it belongs to a dithranol isomer other than the keto or enolate. 


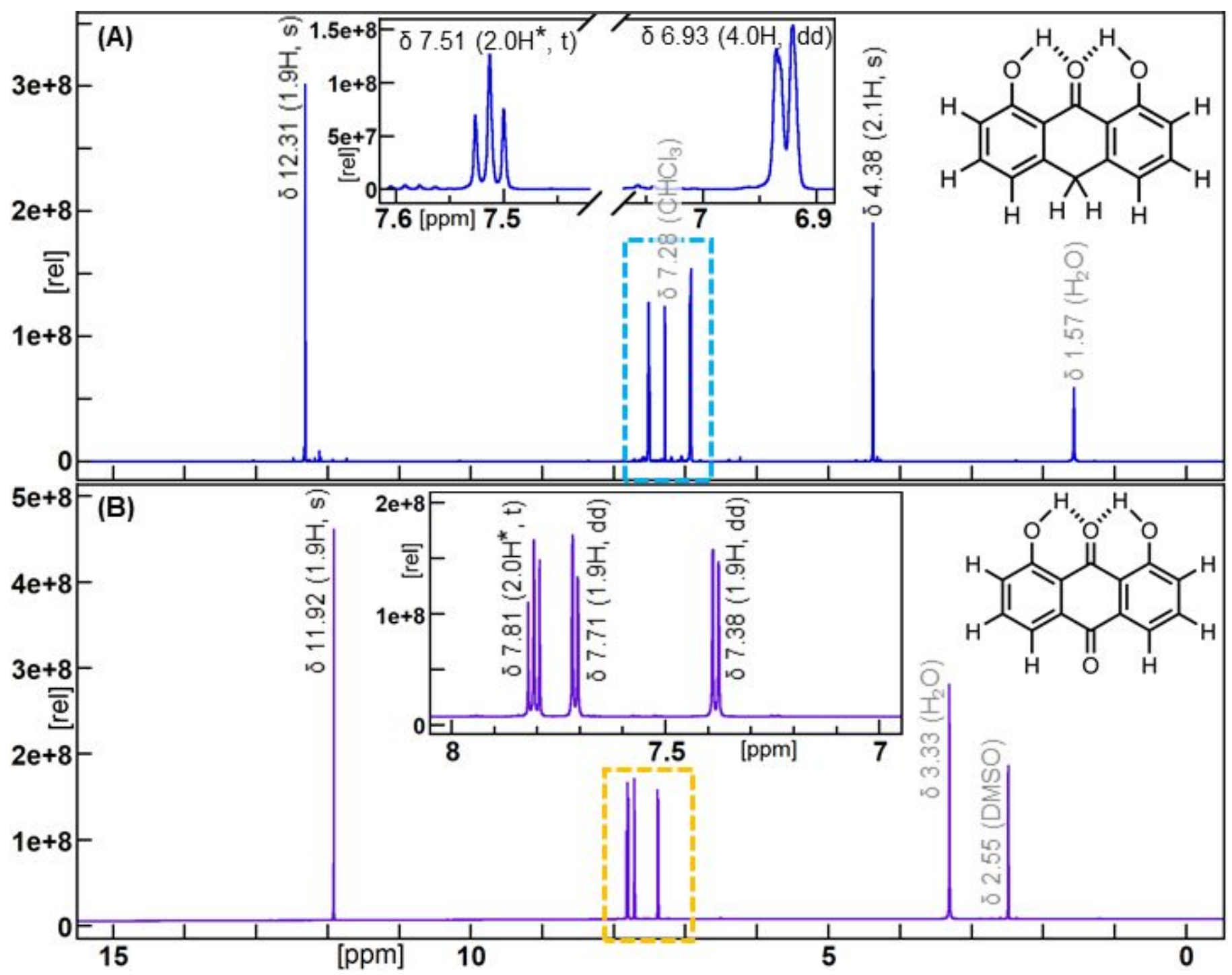

Figure S7. ${ }^{1} \mathrm{H}-\mathrm{NMR}$ spectra of $10 \mathrm{mM}$ dithranol $\left(\mathrm{A} ; \mathrm{CDCl}_{3}, 600 \mathrm{MHz}\right)$ and $18 \mathrm{mM}$ dithranone (B; $\left.\left(\mathrm{CD}_{3}\right)_{2} \mathrm{SO}, 600 \mathrm{MHz}\right)$. In chloroform, the spectrum for dithranol $(\mathrm{A})$ exhibits four sets of peaks, which integrate to a total of ten protons, consistent with the keto isomer of dithranol as shown. The presence of a sharp singlet peak at $12.3 \mathrm{ppm}$, which integrates to two protons, suggests that the two potentially exchangeable hydroxyl protons are not labile, but rather, involved in stable H-bonds ${ }^{4}$ with the carbonyl oxygen on the central ring, as observed in the crystal structure (Scheme S1, 2HB-keto structure). Two protons are represented by a triplet and four by doublet-of-doublet peaks in the region typically associated with aromaticity $(6-8 \mathrm{ppm})$, highlighted in the dashed box and shown on an expanded scale in the inset. An up-field peak at $4.4 \mathrm{ppm}$ represents the two protons of the methylene carbon of the central ring, and their presence is unique to the keto isomers. Dithranol ${ }^{1} \mathrm{H}-\mathrm{NMR}$ summary $\left(\mathrm{CDCl}_{3}, 600 \mathrm{MHz}\right): \delta_{\mathrm{H}} 12.31(2 \mathrm{H}, \mathrm{s}), 7.51\left(2 \mathrm{H}^{*}, \mathrm{t}, J=7.9\right), 6.93(4 \mathrm{H}, \mathrm{dd}, J=1.6,0.5), 4.38$ $(2 \mathrm{H}, \mathrm{s})$. In DMSO, the NMR spectrum of dithranone (B) exhibits four peaks, which integrate to a total of eight protons. The hydroxyl protons are represented by a sharp singlet peak at $11.9 \mathrm{ppm}$ which integrates to two protons, suggesting that these are involved in stable $\mathrm{H}$-bonds ${ }^{4}$ with the carbonyl oxygen on the central ring, as observed in dithranol. Two protons are represented by a triplet and four by doublet-of-doublet peaks in the region typically associated with aromaticity (dashed box, shown on an expanded scale in the inset). Dithranone ${ }^{1} \mathrm{H}-\mathrm{NMR}$ summary $\left(\left(\mathrm{CD}_{3}\right)_{2} \mathrm{SO}, 600 \mathrm{MHz}\right): \delta_{\mathrm{H}} 11.9(2 \mathrm{H}$, s), $7.8(2 \mathrm{H}, \mathrm{t}), 7.7(2 \mathrm{H}, \mathrm{d}), 7.5(2 \mathrm{H}, \mathrm{d})$.

[[ ${ }^{*}$ Integration normalized to this peak. ]] 

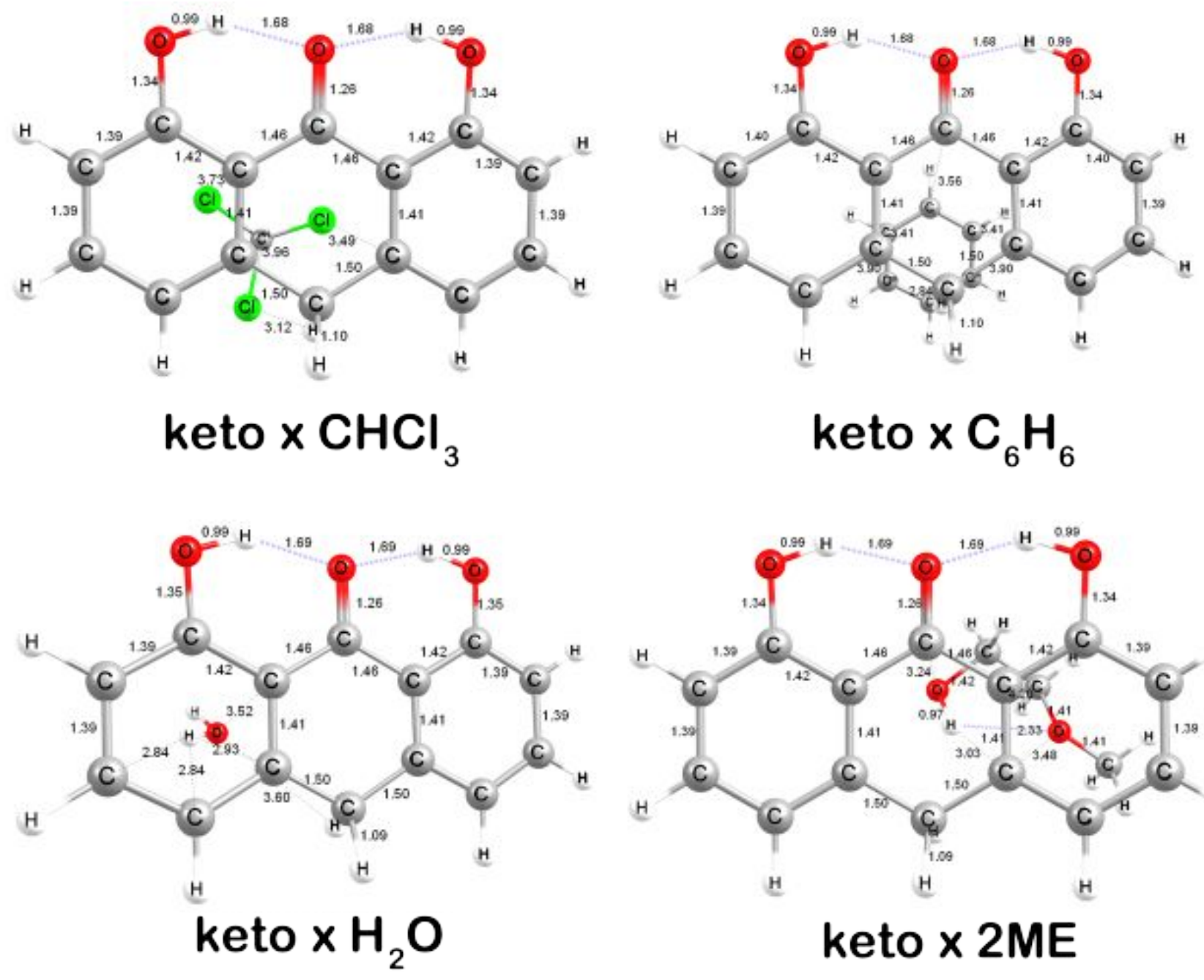

keto $\times \mathrm{C}_{6} \mathrm{H}_{6}$

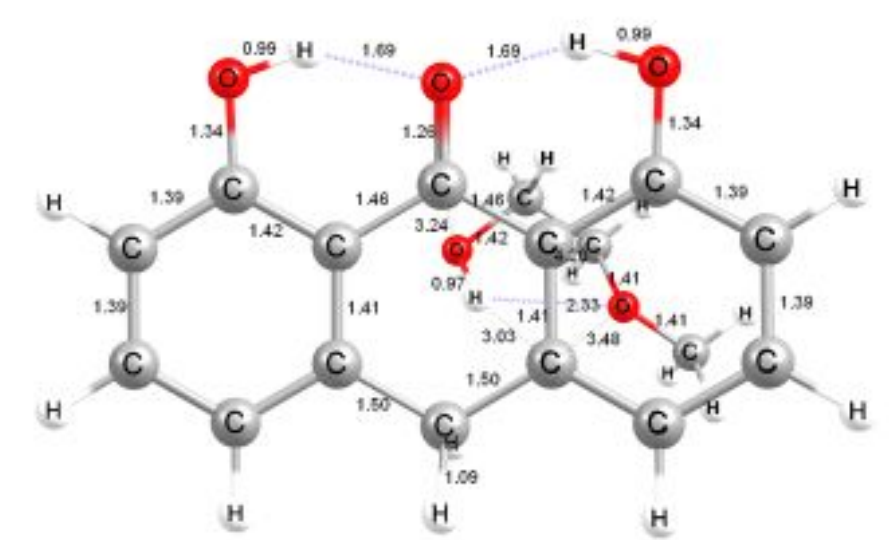

keto $\times 2 \mathrm{ME}$

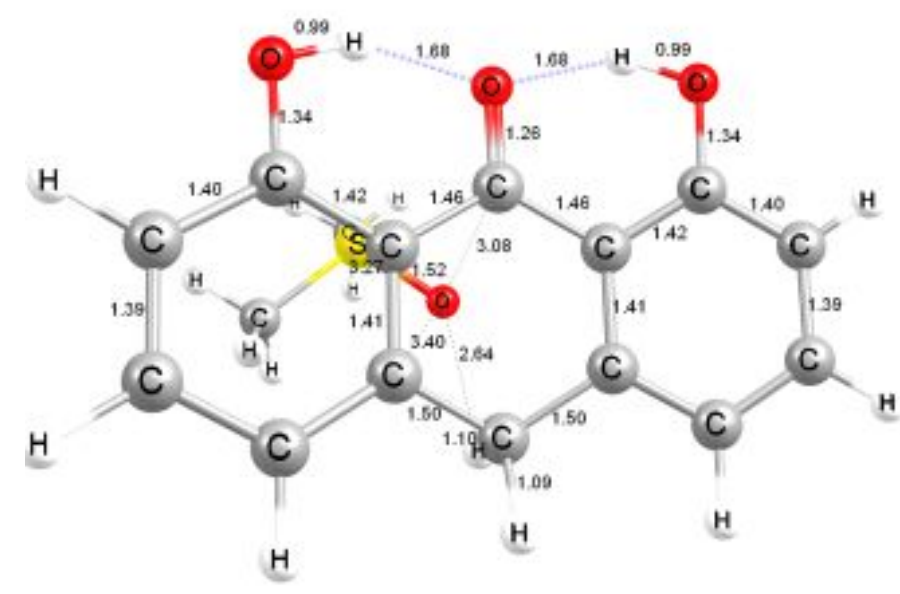

keto $\times$ DMSO

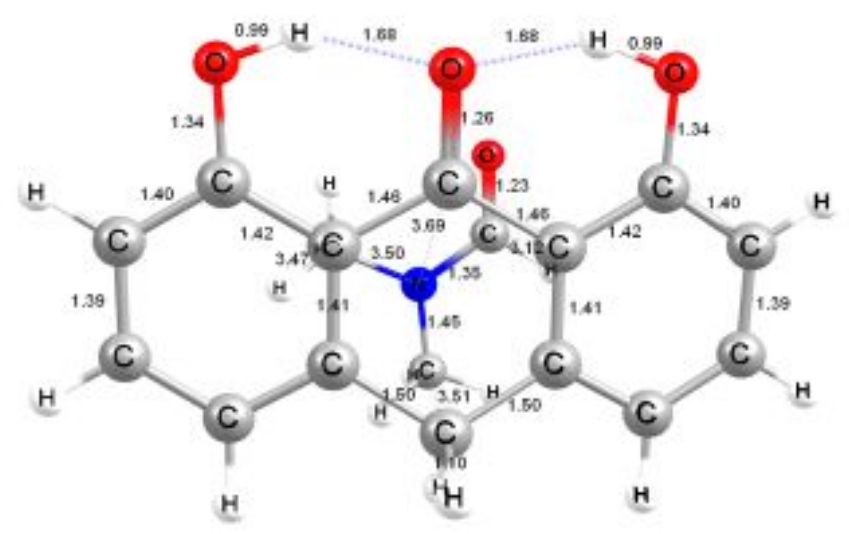

keto $\times$ DMF

Figure S8. Solution phase optimized equilibrium structures (no imaginary normal models) of keto isomers of dithranol with explicit solvents calculated at MN15/6-311++G*/SMD level of theory. 


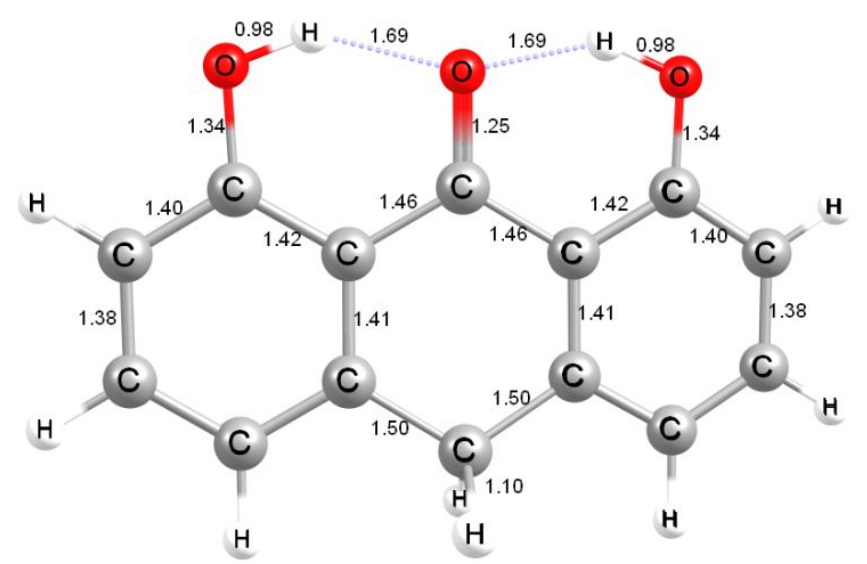

keto

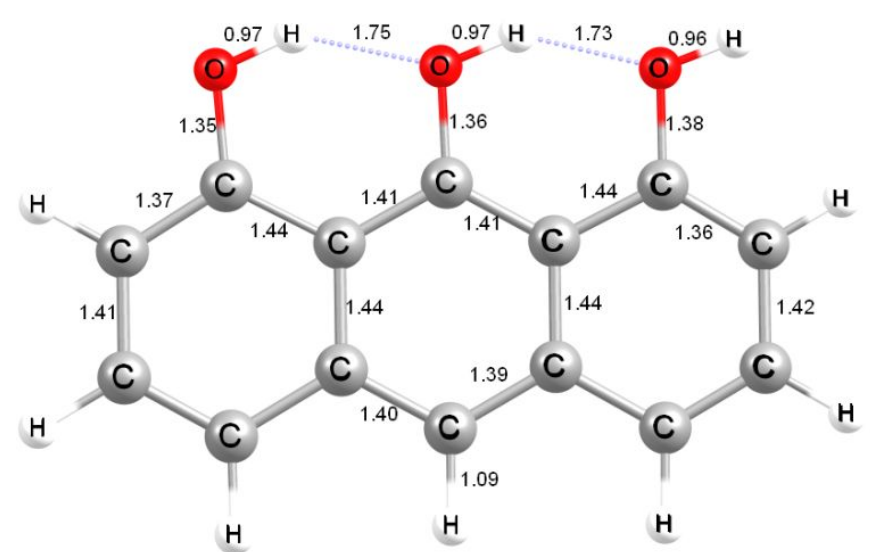

enol

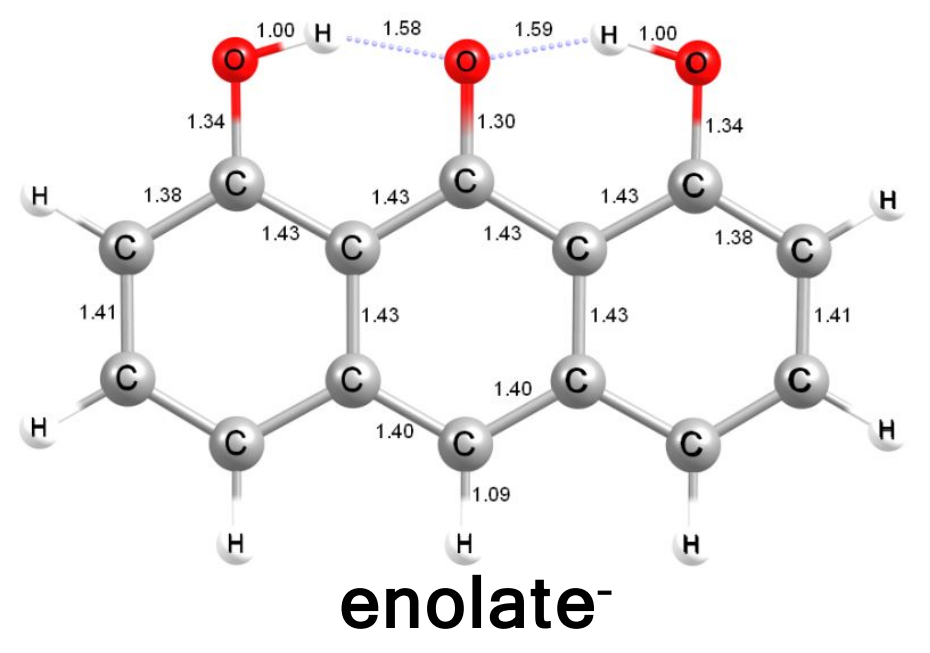

Figure S9. Gas phase optimized equilibrium structures calculated at MN15/6-311++G** level of theory. 


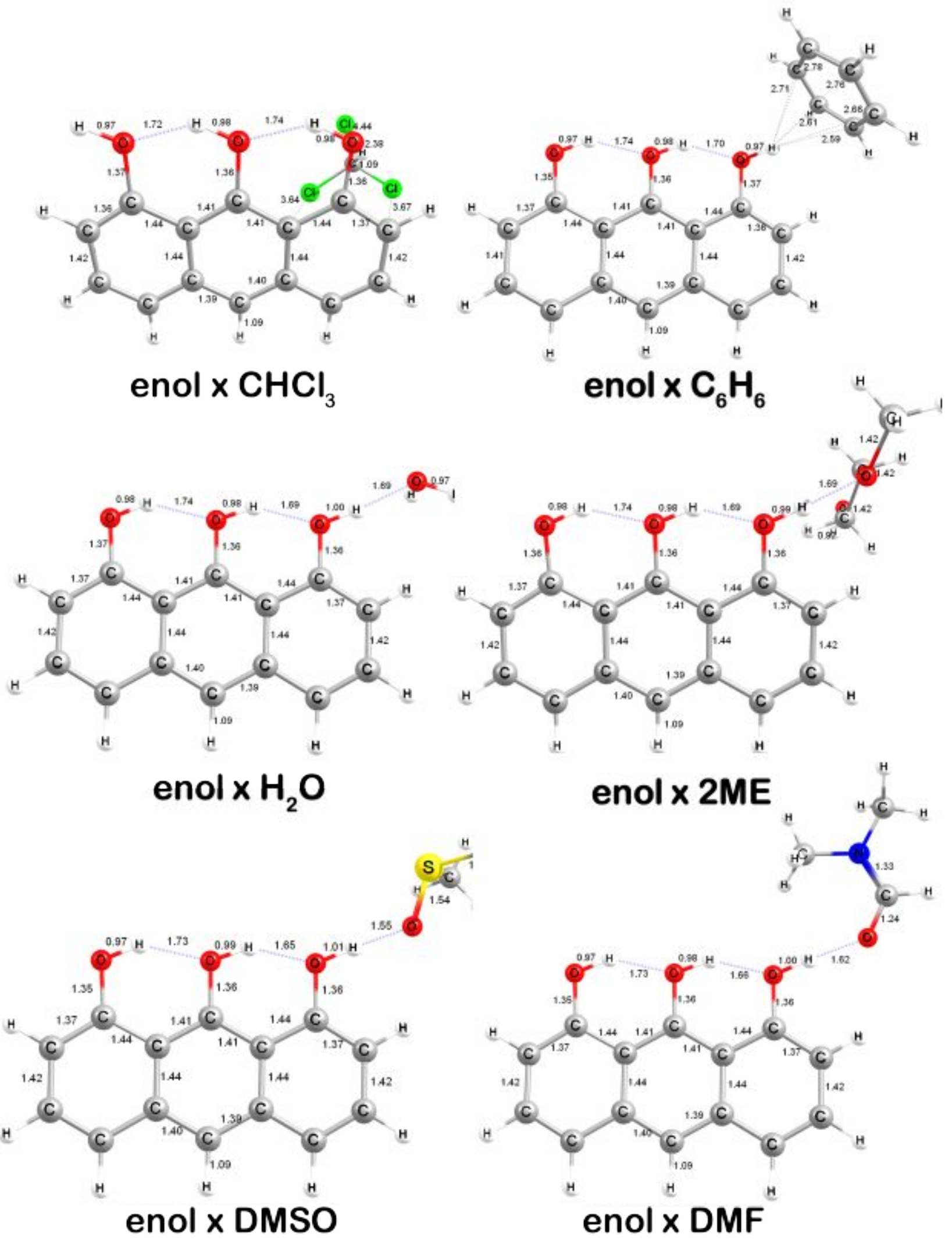

Figure S10. Solution phase optimized equilibrium structures (no imaginary normal models) of enol isomers of dithranol with explicit solvents calculated at MN15/6-311++ $G^{* *} / S M D$ level of theory. 


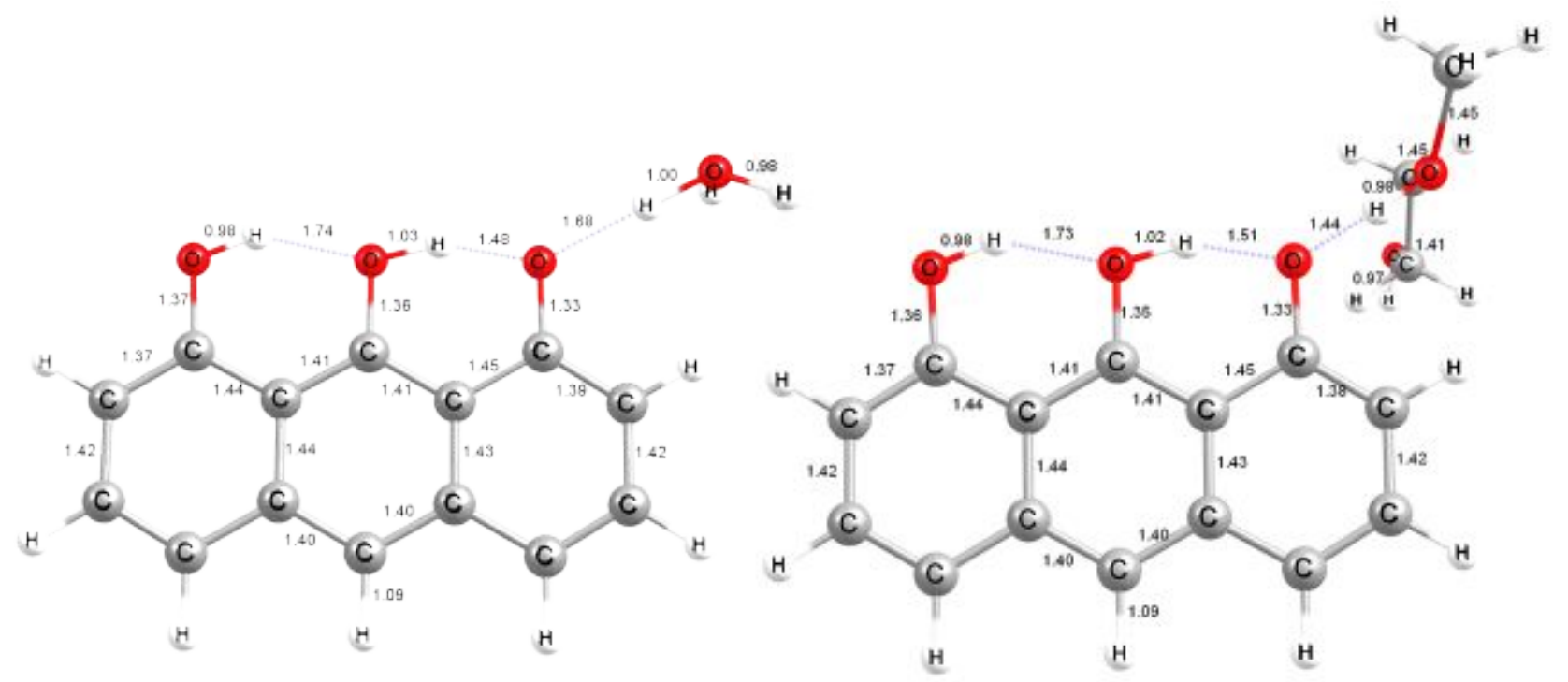

[enolate $^{-} \times \mathrm{H}_{3} \mathrm{O}^{+}$]

\section{[enolate $\left.{ }^{-} \times 2 \mathrm{MEH}^{+}\right]^{\star}$}

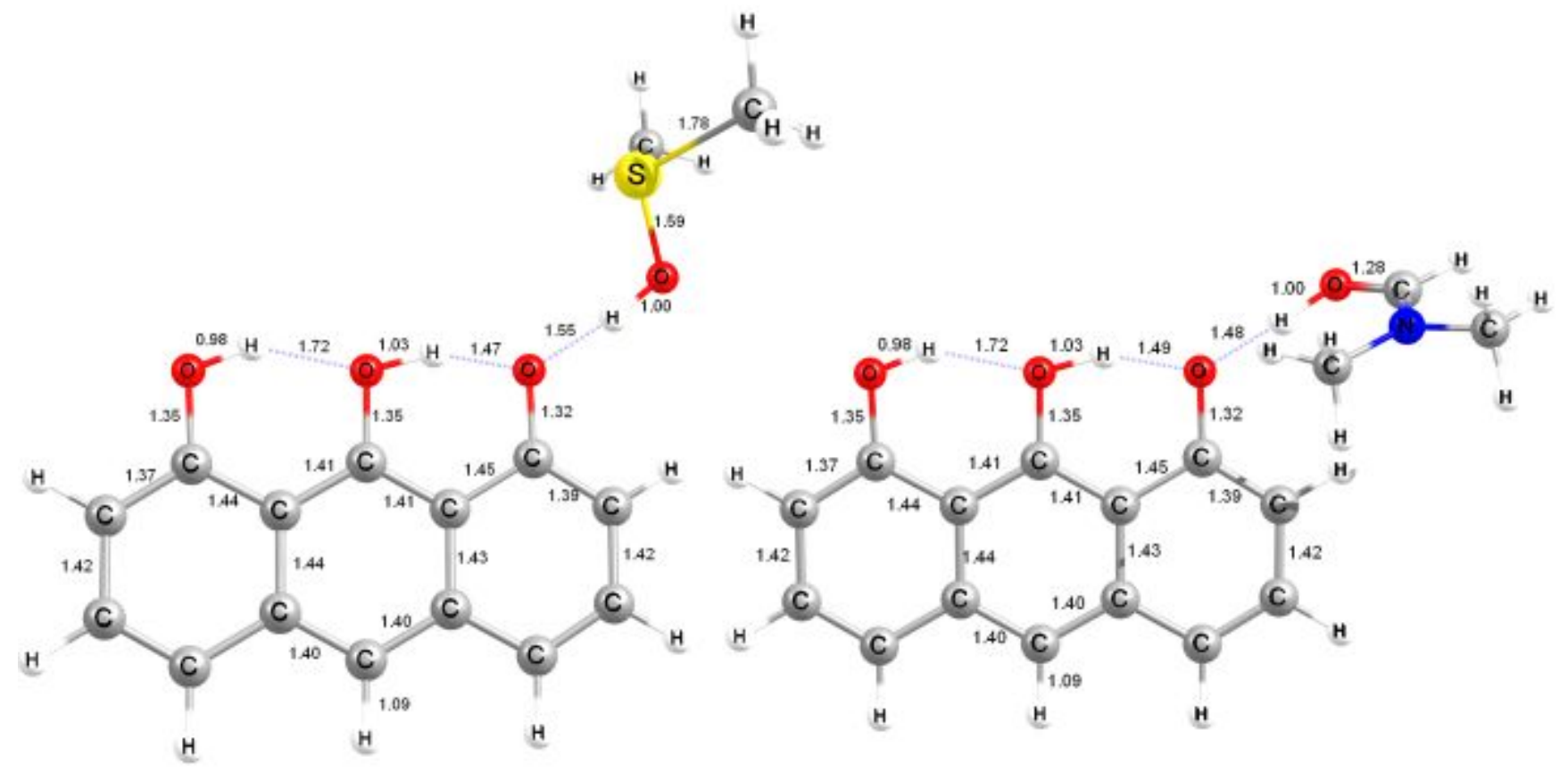

\section{[enolate $\left.^{-} \times \mathrm{DMSOH}^{+}\right]^{\star} \quad\left[\text { enolate }^{-} \times \mathrm{DMFH}^{+}\right]^{\star}$}

Figure S11. Solution phase optimized stationary structures of transient enolate isomers with protonated solvent calculated at MN15/6-311++G**/SMD level of theory. The frozen $\mathrm{O} \cdots \mathrm{H}$ (solvent) distance resulted in a single imaginary normal mode with displacements along the $\mathrm{O}^{-} \cdots \mathrm{H}^{+}-\mathbf{S}$ vector. 

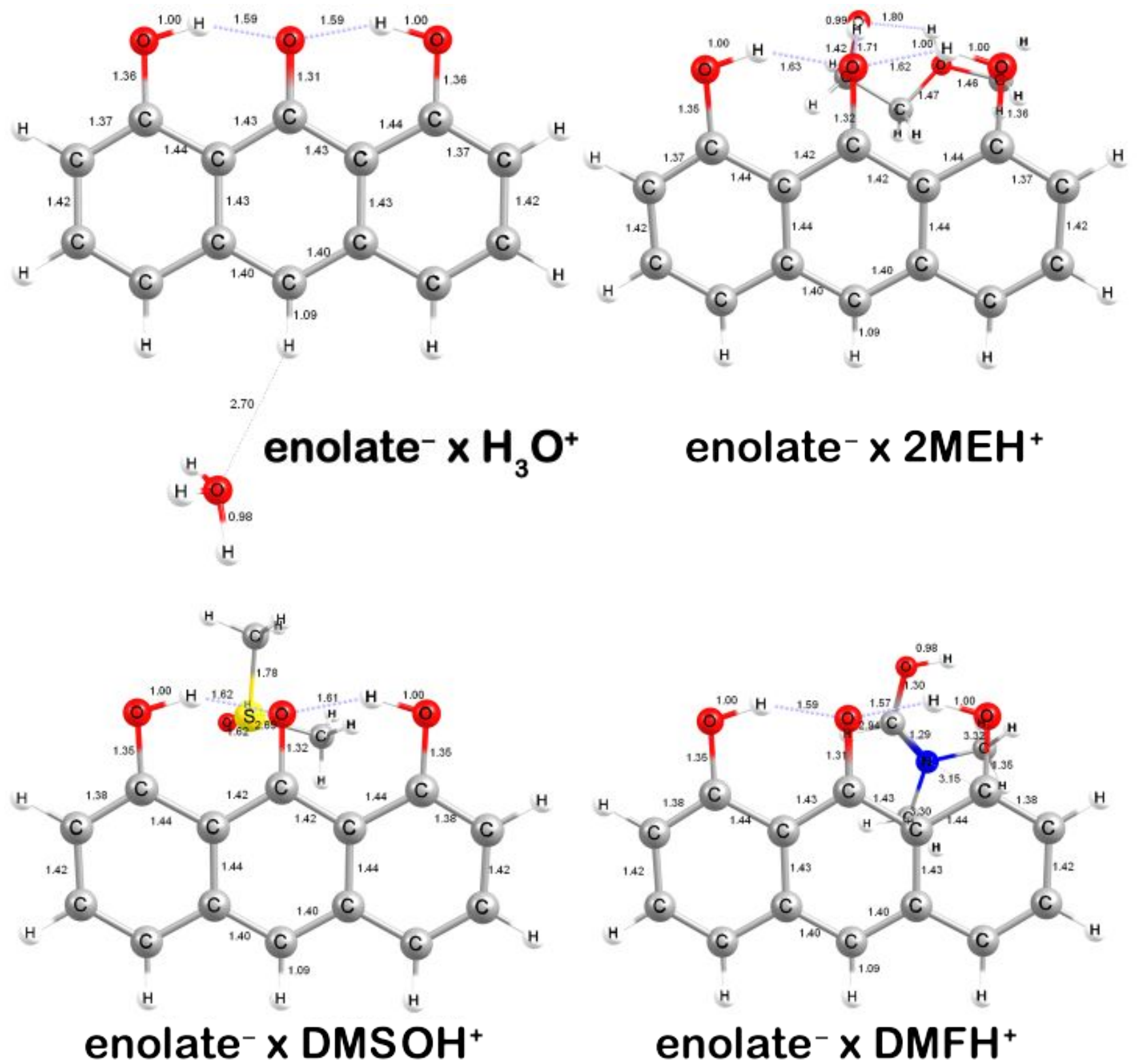

Figure S12. Solution phase optimized equilibrium structures (no imaginary normal models) of enolate form of dithranol with protonated solvent calculated at MN15/6-311++G*/SMD level of theory. 


\section{REFERENCES}

(1) Marenich, A. V.; Kelly, C. P.; Thompson, J. D.; Hawkins, G. D.; Chambers, C. C.; Giesen, D. J.; Winget, P.; Cramer, C. J.; Truhlar, D. G. Minnesota Solvation Database. University of Minnesota: Minneapolis 2012.

(2) Korth, H.-G.; Mulder, P. Anthrone and Related Hydroxyarenes: Tautomerization and Hydrogen Bonding. J. Org. Chem. 2013, 78 (15), 7674-7682. https://doi.org/10.1021/jo401243b.

(3) Whitesides, G. M.; Mammen, M.; Shakhnovich, E. I.; Deutch, J. M. Estimating the Entropic Cost of Self-Assembly of Multiparticle Hydrogen-Bonded Aggregates Based on the Cyanuric Acid Center Dot Melamine Lattice. J. Org. Chem. 1998, 63 (12), 3821-3830.

(4) Sigala, P. A.; Ruben, E. A.; Liu, C. W.; Piccoli, P. M. B.; Hohenstein, E. G.; Martínez, T. J.; Schultz, A. J.; Herschlag, D. Determination of Hydrogen Bond Structure in Water versus Aprotic Environments To Test the Relationship Between Length and Stability. J. Am. Chem. Soc. 2015, 137 (17), 5730-5740. https://doi.org/10.1021/ja512980h.

(5) Miyamoto, H.; Yampolski, Y.; Young, C. L. IUPAC-NIST Solubility Data Series. 103. Oxygen and Ozone in Water, Aqueous Solutions, and Organic Liquids (Supplement to Solubility Data Series Volume 7). J. Phys. Chem. Ref. Data 2014, 43 (3), 033102. https://doi.org/10.1063/1.4883876.

(6) Oxygen and Ozone: Solubility Data Series, Vol 7.; Battino, R., Ed.; Pergamon Press: New York, 1981.

(7) Sato, T.; Hamada, Y.; Sumikawa, M.; Araki, S.; Yamamoto, H. Solubility of Oxygen in Organic Solvents and Calculation of the Hansen Solubility Parameters of Oxygen. Ind. Eng. Chem. Res. 2014, 53 (49), 19331-19337. https://doi.org/10.1021/ie502386t. 